\title{
EXPONENTIAL STABILITY OF TIMOSHENKO BEAM SYSTEM WITH DELAY TERMS IN BOUNDARY FEEDBACKS *
}

\author{
ZHONG-JIE HAN ${ }^{1}$ AND GEN-Qi XU ${ }^{1}$
}

\begin{abstract}
In this paper, the stability of a Timoshenko beam with time delays in the boundary input is studied. The system is fixed at the left end, and at the other end there are feedback controllers, in which time delays exist. We prove that this closed loop system is well-posed. By the complete spectral analysis, we show that there is a sequence of eigenvectors and generalized eigenvectors of the system operator that forms a Riesz basis for the state Hilbert space. Hence the system satisfies the spectrum determined growth condition. Then we conclude the exponential stability of the system under certain conditions. Finally, we give some simulations to support our results.
\end{abstract}

Mathematics Subject Classification. 93D15, 93C20, 49K25, 34H05.

Received March 25, 2009.

Published online March 31, 2010.

\section{INTRODUCTION}

Time delay is a universal phenomenon existing in almost every engineering practices, such as electrical and mechanical engineering, biology and so on (see $[8,14,15])$. Normally, the presence of delays makes the systems less productive, less optimal and less stable $($ see $[2,10])$. However, sometimes it also can play a positive role in the performance of the systems (see $[4,16]$ ).

Due to the effect of time delays, it is necessary to take the delays into account when we discuss the control problem of the systems. For elastic systems, such as wave equations and beam equations, there have been some nice results on control problem with time delays. For example, Liang et al. in [5] introduced the modified Smith predictor to the boundary control of wave equation and Euler-Bernoulli beam equation with a delayed boundary measurement. $\mathrm{Xu}$ et al. in [21,24] considered the Riesz basis property, exact controllability and stability of the string systems with time-delayed boundary feedback control. Mörgul in [9] designed a class of dynamical controllers to robustly stabilize the wave equations against small time delays in the feedback loop. Nicaise and Pignotti in [11] get the exponential stability of the wave equation with boundary or internal distributed delay.

However, at present, there is few result about the stability of Timoshenko beam with time-delayed feedback control. Timoshenko beam model constitutes a weakly coupled system. This weakly coupled property causes that it is usually difficult to consider the control problem for this kind of system. Our aim in this paper is

Keywords and phrases. Timoshenko beam, exponential stability, time delay, Riesz basis, feedback control.

* This research is supported by the Natural Science Foundation of China grant NSFC-60874034.

1 Department of Mathematics, Tianjin University, Tianjin 300072, P.R. China. zjhan@tju.edu.cn 
to discuss the stability of a Timoshenko beam with time delays in the boundary control. What we are interested in is that, under the time-delayed feedback controls, whether the system is still exponentially stable, since it can get the exponential stability without time delays (see [19]).

Let us recall the Timoshenko beam model:

$$
\left\{\begin{array}{l}
\rho w_{t t}(x, t)-k\left(w_{x x}-\varphi_{x}\right)(x, t)=0, \quad x \in(0,1), t>0 \\
I_{\rho} \varphi_{t t}(x, t)-E I \varphi_{x x}(x, t)-k\left(w_{x}-\varphi\right)(x, t)=0, \quad x \in(0,1), t>0
\end{array}\right.
$$

where $\rho, I_{\rho}, E I, k$ are mass density, moment of mass inertia, rigidity coefficient and shear modulus of elastic beam, respectively. For more precise physical meanings of them, see Timoshenko's book [17]. In the sequel, we shall use the abbreviations $w_{t}=\frac{\partial w}{\partial t}$ and $w_{x}=\frac{\partial w}{\partial x}$.

The beam is clamped at the left end, i.e.,

$$
w(0, t)=\varphi(0, t)=0, \quad t>0
$$

The external shearing force and bending moment in which there exist time delays are applied at the right end,

$$
\left\{\begin{array}{l}
k\left(w_{x}-\varphi\right)(1, t)=\mu_{1} v_{1}(t)+\left(1-\mu_{1}\right) v_{1}\left(t-\tau_{1}\right), t>0 \\
E I \varphi_{x}(1, t)=\mu_{2} v_{2}(t)+\left(1-\mu_{2}\right) v_{2}\left(t-\tau_{2}\right), t>0
\end{array}\right.
$$

where $\mu_{i} \in(0,1), i=1,2 . v_{i}\left(t-\tau_{i}\right):=\widetilde{f}_{i}\left(t-\tau_{i}\right), t \in\left(0, \tau_{i}\right)$, and $\widetilde{f}_{i}(\theta), i=1,2$ are the given functions.

The control design (1.3) has been used in [12,24], in which the authors considered 1-d wave systems with time delays. When $\mu_{i}=1, i=1,2$, Xu and Feng [19] and Kim and Renardy [3] have used the feedback control laws

$$
\left\{\begin{array}{l}
v_{1}(t)=-\alpha_{1} w_{t}(1, t), t>0, \alpha_{1}>0 \\
v_{2}(t)=-\alpha_{2} \varphi_{t}(1, t), t>0, \alpha_{2}>0
\end{array}\right.
$$

to exponentially stabilize the system (1.1)-(1.3). However, if $\mu_{i} \neq 1$, the stability of the system (1.1)-(1.3) is unknown. In this paper, we shall discuss this case. Under certain conditions, we prove that the system is still exponentially stable when $\mu_{i} \neq 1$.

In addition, the initial conditions are given as follows:

$$
\begin{cases}w(x, 0)=\widetilde{w}_{0}(x), & w_{t}(x, 0)=\widetilde{w}_{1}(x) \\ \varphi(x, 0)=\widetilde{\varphi}_{0}(x), & \varphi_{t}(x, 0)=\widetilde{\varphi}_{1}(x)\end{cases}
$$

Thus, under the feedback control laws (1.4), we get the following closed loop system:

$$
\left\{\begin{array}{l}
\rho w_{t t}(x, t)-k\left(w_{x x}-\varphi_{x}\right)(x, t)=0, \quad x \in(0,1), t>0, \\
I_{\rho} \varphi_{t t}(x, t)-E I \varphi_{x x}(x, t)-k\left(w_{x}-\varphi\right)(x, t)=0, \quad x \in(0,1), t>0, \\
w(0, t)=\varphi(0, t)=0, t>0, \\
k\left(w_{x}-\varphi\right)(1, t)=-\alpha_{1} \mu_{1} w_{t}(1, t)-\alpha_{1}\left(1-\mu_{1}\right) w_{t}\left(1, t-\tau_{1}\right), t>0, \\
E I \varphi_{x}(1, t)=-\alpha_{2} \mu_{2} \varphi_{t}(1, t)-\alpha_{2}\left(1-\mu_{2}\right) \varphi_{t}\left(1, t-\tau_{2}\right), t>0, \\
w(x, 0)=\widetilde{w}_{0}(x), \quad w_{t}(x, 0)=\widetilde{w}_{1}(x), \quad \varphi(x, 0)=\widetilde{\varphi}_{0}(x), \quad \varphi_{t}(x, 0)=\widetilde{\varphi}_{1}(x), \\
w_{t}\left(1, t-\tau_{1}\right)=-\alpha_{1}^{-1} \widetilde{f}_{1}\left(t-\tau_{1}\right), \varphi_{t}\left(1, t-\tau_{2}\right)=-\alpha_{2}^{-1} \widetilde{f}_{2}\left(t-\tau_{2}\right), \quad t \in\left(0, \tau_{i}\right), i=1,2 .
\end{array}\right.
$$

Set

$$
p(x, t):=w_{t}\left(1, t-x \tau_{1}\right), \quad q(x, t):=\varphi_{t}\left(1, t-x \tau_{2}\right) .
$$


Then the system (1.6) is equivalent to

$$
\left\{\begin{array}{l}
\rho w_{t t}(x, t)-k\left(w_{x x}-\varphi_{x}\right)(x, t)=0, \quad x \in(0,1), t>0 \\
I_{\rho} \varphi_{t t}(x, t)-E I \varphi_{x x}(x, t)-k\left(w_{x}-\varphi\right)(x, t)=0, \quad x \in(0,1), t>0, \\
\tau_{1} p_{t}(x, t)+p_{x}(x, t)=0, \quad \tau_{2} q_{t}(x, t)+q_{x}(x, t)=0, \quad x \in(0,1), t>0 \\
w(0, t)=\varphi(0, t)=0, \quad t>0, \\
p(0, t)=w_{t}(1, t), \quad q(0, t)=\varphi_{t}(1, t), \quad t>0, \\
k\left(w_{x}-\varphi\right)(1, t)=-\alpha_{1} \mu_{1} w_{t}(1, t)-\alpha_{1}\left(1-\mu_{1}\right) p(1, t), \quad t>0, \\
E I \varphi_{x}(1, t)=-\alpha_{2} \mu_{2} \varphi_{t}(1, t)-\alpha_{2}\left(1-\mu_{2}\right) q(1, t), \quad t>0, \\
w(x, 0)=\widetilde{w}_{0}(x), \quad w_{t}(x, 0)=\widetilde{w}_{1}(x), \quad \varphi(x, 0)=\widetilde{\varphi}_{0}(x), \quad \varphi_{t}(x, 0)=\widetilde{\varphi}_{1}(x), \\
p(x, 0)=-\alpha_{1}^{-1} \widetilde{f}_{1}\left(-x \tau_{1}\right), q(x, 0)=-\alpha_{2}^{-1} \widetilde{f}_{2}\left(-x \tau_{2}\right), \quad x \in(0,1) .
\end{array}\right.
$$

Note that this system is too complex to construct a Lyapunov function or a multiplier to analyze its stability. Herein we mainly employ the Riesz basis approach. For a vibrating system, the Riesz basis generation is the most profound result. It forms a basis not only for the expansion of the solution in terms of the (generalized) eigenvectors of the system but also for the spectrum determined growth condition. Since the spectral analysis is a key to develop Riesz basis property, we shall have the complete spectral analysis for the system (1.7). Based on the distribution of the spectrum of this system, we prove that there exists a sequence of (generalized) eigenvectors of the system (1.7) that forms a Riesz basis for the state space. Hence, the spectrum determined growth condition holds, i.e., the growth order of the system is determined via its spectral bound. Therefore, we conclude the exponential stability by showing that the imaginary axis is not an asymptote of the spectrum of the system operator.

The remaining part of this paper is organized as follows. In Section 2, the system (1.7) is formulated in a Hilbert space setting and the well-posedness of the system is proved. Section 3 is devoted to the spectral analysis of the system. In Section 4, the completeness as well as the Riesz basis property of the (generalized) eigenvectors of the system operator is presented. The exponential stability of the system is discussed in Section 5. Finally, in Section 6, some simulations are given to support our results.

\section{WELL-POSEDNESS OF THE SYSTEM}

In this section, we shall study the well-posedness of the closed loop system (1.7). To this end, we formulate this system in an appropriate Hilbert space setting.

Set

$$
V^{k}(0,1):=\left\{f \in H^{k}(0,1) \mid f(0)=0\right\},
$$

where $H^{k}(0,1)$ is the usual Sobolev space of order $k$.

Let the state space be

$$
\mathcal{H}:=V^{1}(0,1) \times L^{2}(0,1) \times L^{2}(0,1) \times V^{1}(0,1) \times L^{2}(0,1) \times L^{2}(0,1)
$$

endowed with an inner product: for $W_{i}=\left(w_{i}, z_{i}, p_{i}, \varphi_{i}, \psi_{i}, q_{i}\right) \in \mathcal{H}, i=1,2$

$$
\begin{aligned}
\left(W_{1}, W_{2}\right)_{\mathcal{H}}:= & \int_{0}^{1} k\left(w_{1, x}-\varphi_{1}\right) \overline{\left(w_{2, x}-\varphi_{2}\right)} \mathrm{d} x+\int_{0}^{1} E I \varphi_{1, x} \overline{\varphi_{2, x}} \mathrm{~d} x+\int_{0}^{1} \rho z_{1} \overline{z_{2}} \mathrm{~d} x \\
& +\int_{0}^{1} I_{\rho} \psi_{1} \bar{\psi}_{2} \mathrm{~d} x+\int_{0}^{1} p_{1} \overline{p_{2}} \mathrm{~d} x+\int_{0}^{1} q_{1} \overline{q_{2}} \mathrm{~d} x
\end{aligned}
$$

where $k, \rho, I_{\rho}, E I$ are all positive parameters in the system (1.7). A direct verification shows that $\left(\mathcal{H},\|\cdot\|_{\mathcal{H}}\right)$ is a Hilbert space. 
We define the operator $\mathcal{A}$ in $\mathcal{H}$ as follows

$$
\mathcal{A}(w, z, p, \varphi, \psi, q)^{\tau}=\left(\begin{array}{c}
z \\
\rho^{-1} k\left(w_{x x}-\varphi_{x}\right) \\
-\tau_{1}^{-1} p_{x} \\
\psi \\
I_{\rho}^{-1} E I \varphi_{x x}+I_{\rho}^{-1} k\left(w_{x}-\varphi\right) \\
-\tau_{2}^{-1} q_{x}
\end{array}\right)
$$

with domain

$$
\begin{aligned}
& \mathcal{D}(\mathcal{A})=\left\{(w, z, p, \varphi, \psi, q)^{\tau} \in V^{2}(0,1) \times V^{1}(0,1) \times H^{1}(0,1) \times V^{2}(0,1) \times V^{1}(0,1) \times H^{1}(0,1)\right. \\
& \left.k\left(w_{x}(1)-\varphi(1)\right)=-\alpha_{1} \mu_{1} z(1)-\alpha_{1}\left(1-\mu_{1}\right) p(1), z(1)=p(0)\right\} .
\end{aligned}
$$

Consequently, the problem (1.7) can be rewritten as an evolutionary equation in $\mathcal{H}$ :

$$
\left\{\begin{array}{l}
\frac{\mathrm{d}}{\mathrm{d} t} W(t)=\mathcal{A} W(t), \quad t>0, \\
W(0)=W_{0}
\end{array}\right.
$$

where $W(t)=(w(x, t), \dot{w}(x, t), p(x, t), \varphi(x, t), \dot{\varphi}(x, t), q(x, t))^{\tau}$ and

$$
W_{0}=\left(\widetilde{w}_{0}, \widetilde{w}_{1},-\alpha_{1}^{-1} \widetilde{f}_{1}, \widetilde{\varphi}_{0}, \widetilde{\varphi}_{1},-\alpha_{2}^{-1} \widetilde{f}_{2}\right)^{\tau}
$$

We have the following result:

Lemma 2.1. Let $\mathcal{A}$ and $\mathcal{H}$ be defined as before. Then for any $\alpha_{i}, \mu_{i} \in \mathbb{R}_{+}, i=1,2,0 \in \rho(\mathcal{A})$ and $\mathcal{A}^{-1}$ is compact on $\mathcal{H}$.

Proof. Clearly, $\mathcal{D}(\mathcal{A})$ is dense in $\mathcal{H}$. Let $\mu_{i}, \alpha_{i} \in \mathbb{R}_{+}, i=1,2$ be given. For any $F=\left(f_{1}, f_{2}, f_{3}, f_{4}, f_{5}, f_{6}\right) \in \mathcal{H}$, we consider the solvability of equation $\mathcal{A} W=F$, where $W=(w, z, p, \varphi, \psi, q)^{\tau} \in \mathcal{D}(\mathcal{A})$, i.e.,

$$
\left\{\begin{array}{l}
z(x)=f_{1}(x), \quad \rho^{-1} k\left(w_{x x}-\varphi_{x}\right)(x)=f_{2}(x) \\
\tau_{1}^{-1} p_{x}(x)=-f_{3}(x), \quad \psi(x)=f_{4}(x) \\
I_{\rho}^{-1} E I \varphi_{x x}(x)+I_{\rho}^{-1} k\left(w_{x}-\varphi\right)(x)=f_{5}(x), \quad \tau_{2}^{-1} q_{x}(x)=-f_{6}(x)
\end{array}\right.
$$

with the boundary conditions

$$
\left\{\begin{array}{l}
w(0)=\varphi(0)=0, \quad p(0)=z(1), \quad q(0)=\psi(1) \\
k\left(w_{x}(1)-\varphi(1)\right)=-\alpha_{1} \mu_{1} z(1)-\alpha_{1}\left(1-\mu_{1}\right) p(1) \\
E I \varphi_{x}(1)=-\alpha_{2} \mu_{2} \psi(1)-\alpha_{2}\left(1-\mu_{2}\right) q(1)
\end{array}\right.
$$

Solving the ordinary differential equations $(2.5)-(2.6)$ yields

$$
\left\{\begin{aligned}
p(x)= & f_{1}(1)-\tau_{1} \int_{0}^{x} f_{3}(s) \mathrm{d} s, \quad q(x)=f_{4}(1)-\tau_{2} \int_{0}^{x} f_{6}(s) \mathrm{d} s \\
\varphi(x)=- & E I^{-1} \alpha_{2} f_{4}(1) x+E I^{-1} \alpha_{2}\left(1-\mu_{2}\right) \tau_{2} x \int_{0}^{1} f_{6}(s) \mathrm{d} s-E I^{-1} \int_{0}^{x} \int_{s}^{1}\left(I_{\rho} f_{5}(r)-G_{1}(r)\right) \mathrm{d} r \mathrm{~d} s \\
w(x)=- & E I^{-1} \alpha_{2} f_{4}(1) \frac{x^{2}}{2}+E I^{-1} \alpha_{2}\left(1-\mu_{2}\right) \tau_{2} \frac{x^{2}}{2} \int_{0}^{1} f_{6}(s) \mathrm{d} s-E I^{-1} \int_{0}^{x} \int_{0}^{s} \int_{r}^{1}\left(I_{\rho} f_{5}(\xi)-G_{1}(\xi)\right) \mathrm{d} \xi \mathrm{d} r \mathrm{~d} s \\
& \quad+k^{-1} \int_{0}^{x} G_{1}(s) \mathrm{d} s
\end{aligned}\right.
$$


where

$$
G_{1}(x):=\left[-\alpha_{1} f_{1}(1)+\alpha_{1} \tau_{1}\left(1-\mu_{1}\right) \int_{0}^{1} f_{3}(x) \mathrm{d} x\right]-\int_{x}^{1} \rho f_{2}(s) \mathrm{d} s .
$$

For these $p(x), q(x), \varphi(x), w(x)$, which are given by $(2.7)$, we see that $W=\left(w, f_{1}, p, \varphi, f_{4}, q\right) \in \mathcal{D}(\mathcal{A}) \subset \mathcal{H}$ and $\mathcal{A}\left(w, f_{1}, p, \varphi, f_{4}, q\right)=F$. Hence $\mathcal{A}^{-1}$ exists. Thus, the Sobolev's Embedding Theorem implies that $\mathcal{A}^{-1}$ is a compact operator on $\mathcal{H}$.

As a direct consequence of Lemma 2.1, we have the following result.

Corollary 2.1. Let $\mathcal{A}$ and $\mathcal{H}$ be defined as before. Then the spectrum of $\mathcal{A}$ only consists of isolated eigenvalues of finite multiplicity.

Suppose that

$$
\mu_{i} \geq \frac{1}{2}, \quad i=1,2 .
$$

Under this condition, we shall show that $\mathcal{A}$ is dissipative in $\mathcal{H}$. To this end, we choose the positive real constants $\eta_{i}, i=1,2$ such that

$$
\tau_{i}\left(1-\mu_{i}\right) \alpha_{i} \leq \eta_{i} \leq \tau_{i}\left(3 \mu_{i}-1\right) \alpha_{i}, i=1,2 .
$$

These constants $\eta_{i}, i=1,2$ exist due to the condition (2.8). Then we introduce a new inner product in $\mathcal{H}$ : for $W_{j}=\left(w_{j}, z_{j}, p_{j}, \varphi_{j}, \psi_{j}, q_{j}\right)^{\tau} \in \mathcal{H}, j=1,2$,

$$
\begin{aligned}
\left(W_{1}, W_{2}\right)_{1}= & \int_{0}^{1} k\left(w_{1, x}-\varphi_{1}\right) \overline{\left(w_{2, x}-\varphi_{2}\right)} \mathrm{d} x+\int_{0}^{1} E I \varphi_{1, x} \overline{\varphi_{2, x}} \mathrm{~d} x+\int_{0}^{1} \rho z_{1} \overline{z_{2}} \mathrm{~d} x \\
& +\int_{0}^{1} I_{\rho} \psi_{1} \bar{\psi}_{2} \mathrm{~d} x+\int_{0}^{1} \eta_{1} p_{1} \overline{p_{2}} \mathrm{~d} x+\int_{0}^{1} \eta_{2} q_{1} \overline{q_{2}} \mathrm{~d} x .
\end{aligned}
$$

It is easy to verify that $\left(W_{1}, W_{2}\right)_{1}$ is equivalent to the inner product $\left(W_{1}, W_{2}\right)_{\mathcal{H}}$. Then we have the following result:

Lemma 2.2. Let $\mathcal{A}$ and $\mathcal{H}$ be defined as before and the condition (2.8) be fulfilled. Then $\mathcal{A}$ is dissipative in $\left(\mathcal{H},\|\cdot\|_{1}\right)$.

Proof. For any real $W=(w, z, p, \varphi, \psi, q)^{\tau} \in \mathcal{D}(\mathcal{A})$, we have

$$
\begin{aligned}
(\mathcal{A} W, W)_{1}= & \left.k\left(w_{x}-\varphi\right) z\right|_{0} ^{1}+\left.E I \varphi_{x} \psi\right|_{0} ^{1}-\left.\frac{\tau_{1}^{-1} \eta_{1}}{2} p^{2}\right|_{0} ^{1}-\left.\frac{\tau_{2}^{-1} \eta_{2}}{2} q^{2}\right|_{0} ^{1} \\
= & -\left(\alpha_{1} \mu_{1}-\frac{\eta_{1}}{2 \tau_{1}}\right)(p(0))^{2}-\left(\alpha_{2} \mu_{2}-\frac{\eta_{2}}{2 \tau_{2}}\right)(q(0))^{2}-\frac{\eta_{1}}{2 \tau_{1}}(p(1))^{2}-\frac{\eta_{2}}{2 \tau_{2}}(q(1))^{2} \\
& -\alpha_{1}\left(1-\mu_{1}\right) p(0) p(1)-\alpha_{2}\left(1-\mu_{2}\right) q(0) q(1) \\
\leq & -\left[\alpha_{1} \mu_{1}-\frac{\eta_{1}}{2 \tau_{1}}-\frac{\alpha_{1}\left(1-\mu_{1}\right)}{2}\right](p(0))^{2}-\left[\alpha_{2} \mu_{2}-\frac{\eta_{2}}{2 \tau_{2}}-\frac{\alpha_{2}\left(1-\mu_{2}\right)}{2}\right](q(0))^{2} \\
& -\left[\frac{\eta_{1}}{2 \tau_{1}}-\frac{\alpha_{1}\left(1-\mu_{1}\right)}{2}\right](p(1))^{2}-\left[\frac{\eta_{2}}{2 \tau_{2}}-\frac{\alpha_{2}\left(1-\mu_{2}\right)}{2}\right](q(1))^{2}
\end{aligned}
$$

with

$$
-\alpha_{1}\left(1-\mu_{1}\right) p(0) p(1) \leq \frac{\alpha_{1}\left(1-\mu_{1}\right)}{2}\left[(p(0))^{2}+(p(1))^{2}\right],-\alpha_{2}\left(1-\mu_{2}\right) q(0) q(1) \leq \frac{\alpha_{2}\left(1-\mu_{2}\right)}{2}\left[(q(0))^{2}+(q(1))^{2}\right] .
$$

From (2.9), we get that $\alpha_{1} \mu_{1}-\frac{\eta_{1}}{2 \tau_{1}}-\frac{\alpha_{1}\left(1-\mu_{1}\right)}{2}, \frac{\eta_{1}}{2 \tau_{1}}-\frac{\alpha_{1}\left(1-\mu_{1}\right)}{2}, \alpha_{2} \mu_{2}-\frac{\eta_{2}}{2 \tau_{2}}-\frac{\alpha_{2}\left(1-\mu_{2}\right)}{2}$ and $\frac{\eta_{2}}{2 \tau_{2}}-\frac{\alpha_{2}\left(1-\mu_{2}\right)}{2}$ are all nonnegative. Hence $(\mathcal{A} W, W)_{1} \leq 0$, which implies that $\mathcal{A}$ is dissipative in $\left(\mathcal{H},\|\cdot\|_{1}\right)$. 
By Lemmas 2.1 and 2.2, the Lumer-Phillips Theorem (see Pazy [13]) asserts the following result.

Theorem 2.1. Let $\mathcal{A}$ and $\mathcal{H}$ be defined as before and the condition $(2.8)$ be fulfilled. Then $\mathcal{A}$ generates a $C_{0}$ semigroup $T(t)$ of contractions on $\mathcal{H}$. Hence the closed loop system (1.7) is well-posed.

\section{Spectral analysis of $\mathcal{A}$}

In this section, we shall discuss the asymptotic distribution of the spectrum of $\mathcal{A}$. From Corollary 2.1, we have known that the spectrum of $\mathcal{A}$ only consists of isolated eigenvalues of finite multiplicity. So $\sigma(\mathcal{A})=\sigma_{p}(\mathcal{A})$. Thus we only need to discuss the eigenvalues of $\mathcal{A}$. Let $\lambda \in \sigma_{p}(\mathcal{A}),(w, z, p, \varphi, \psi, q)^{\tau} \in \mathcal{D}(\mathcal{A})$ be an eigenvector of $\mathcal{A}$ corresponding to $\lambda$. Then $z(x)=\lambda w(x)$ and $\psi(x)=\lambda \varphi(x)$. Thus, $w(x), \varphi(x), p$ and $q$ satisfy the following differential equations

$$
\left\{\begin{array}{l}
\rho \lambda^{2} w-k\left(w_{x x}-\varphi_{x}\right)=0, \quad x \in(0,1), \\
I_{\rho} \lambda^{2} \varphi-E I \varphi_{x x}-k\left(w_{x}-\varphi\right)=0, \quad x \in(0,1), \\
\tau_{1} \lambda p+p_{x}=0, \quad \tau_{2} \lambda q+q_{x}=0, \quad x \in(0,1), \\
w(0)=\varphi(0)=0, \quad p(0)=\lambda w(1), \quad q(0)=\lambda \varphi(1), \\
k\left(w_{x}-\varphi\right)(1)=-\alpha_{1} \mu_{1} \lambda w(1)-\alpha_{1}\left(1-\mu_{1}\right) p(1), \\
E I \varphi_{x}(1)=-\alpha_{2} \mu_{2} \lambda \varphi(1)-\alpha_{2}\left(1-\mu_{2}\right) q(1) .
\end{array}\right.
$$

\subsection{Fundamental matrix solution}

In order to calculate the eigenvalues of $\mathcal{A}$, we shall find the fundamental matrix solution to the following differential equation:

Setting

$$
\left\{\begin{array}{l}
\rho \lambda^{2} w-k\left(w_{x x}-\varphi_{x}\right)=0, \quad x \in(0,1) \\
I_{\rho} \lambda^{2} \varphi-E I \varphi_{x x}-k\left(w_{x}-\varphi\right)=0, \quad x \in(0,1)
\end{array}\right.
$$

$$
Y_{1}:=(w, \varphi)^{\tau}, Y_{2}:=\frac{Y_{1}^{\prime}}{\lambda}, Y:=\left[\begin{array}{c}
Y_{1} \\
Y_{2}
\end{array}\right]
$$

we have

$$
\frac{\mathrm{d} Y}{\mathrm{~d} x}=\widetilde{A} Y, \quad \widetilde{A}:=\left[\begin{array}{cccc}
0 & 0 & \lambda & 0 \\
0 & 0 & 0 & \lambda \\
\frac{\rho}{k} \lambda & 0 & 0 & 1 \\
0 & \frac{I_{\rho}}{E I} \lambda+\frac{k}{E I \lambda} & -\frac{k}{E I} & 0
\end{array}\right] .
$$

Under the following transformation:

$$
Y(x)=T_{0} Z(x), \quad T_{0}:=\left[\begin{array}{cccc}
1 & 0 & 1 & 0 \\
0 & 1 & 0 & 1 \\
\sqrt{\frac{\rho}{k}} & 0 & -\sqrt{\frac{\rho}{k}} & 0 \\
0 & \sqrt{\frac{I_{\rho}}{E I}} & 0 & -\sqrt{\frac{I_{\rho}}{E I}}
\end{array}\right]
$$

(3.4) is transformed into the following equation:

$$
\frac{\mathrm{d} Z(x)}{\mathrm{d} x}=T_{0}^{-1} \widetilde{A} T_{0} Z(x) .
$$

A direct calculation leads to $T_{0}^{-1} \widetilde{A} T_{0}:=\lambda \widetilde{\Lambda}_{1}+\widetilde{\Lambda}_{0}+\frac{1}{\lambda} \widetilde{\Lambda}_{-1}$, where

$$
\widetilde{\Lambda}_{1}:=\left(\widetilde{\Lambda}_{1}^{i j}\right)_{4 \times 4}=\left[\begin{array}{cccc}
\sqrt{\frac{\rho}{k}} & 0 & 0 & 0 \\
0 & \sqrt{\frac{I_{\rho}}{E I}} & 0 & 0 \\
0 & 0 & -\sqrt{\frac{\rho}{k}} & 0 \\
0 & 0 & 0 & -\sqrt{\frac{I_{\rho}}{E I}}
\end{array}\right], \quad \widetilde{\Lambda}_{-1}:=\left(\widetilde{\Lambda}_{-1}^{i j}\right)_{4 \times 4}=\left[\begin{array}{cccc}
0 & 0 & 0 & 0 \\
0 & \frac{k}{2 \sqrt{I_{\rho} E I}} & 0 & \frac{k}{2 \sqrt{I_{\rho} E I}} \\
0 & 0 & 0 & 0 \\
0 & -\frac{k}{2 \sqrt{I_{\rho} E I}} & 0 & -\frac{k}{2 \sqrt{I_{\rho} E I}}
\end{array}\right]
$$


and

Therefore, we have

$$
\widetilde{\Lambda}_{0}:=\left(\widetilde{\Lambda}_{0}^{i j}\right)_{4 \times 4}=\left[\begin{array}{cccc}
0 & \frac{\sqrt{I_{\rho} k}}{2 \sqrt{\rho E I}} & 0 & -\frac{\sqrt{I_{\rho} k}}{2 \sqrt{\rho E I}} \\
-\frac{\sqrt{k \rho}}{2 \sqrt{I_{\rho} E I}} & 0 & \frac{\sqrt{k \rho}}{2 \sqrt{I_{\rho} E I}} & 0 \\
0 & -\frac{\sqrt{I_{\rho} k}}{2 \sqrt{\rho E I}} & 0 & \frac{\sqrt{I_{\rho} k}}{2 \sqrt{\rho E I}} \\
\frac{\sqrt{k \rho}}{2 \sqrt{I_{\rho} E I}} & 0 & -\frac{\sqrt{k \rho}}{2 \sqrt{I_{\rho} E I}} & 0
\end{array}\right] .
$$

$$
\frac{\mathrm{d} Z(x)}{\mathrm{d} x}=\left[\lambda \widetilde{\Lambda}_{1}+\widetilde{\Lambda}_{0}+\frac{1}{\lambda} \widetilde{\Lambda}_{-1}\right] Z(x)
$$

Set

where

$$
Z(x, \lambda):=\sum_{k=0}^{\infty} \frac{P_{-k}(x)}{\lambda^{k}} E(x, \lambda)
$$

$$
E(x, \lambda):=\exp \left(x \lambda \widetilde{\Lambda}_{1}\right)
$$

We shall identify $P_{-k}, k=0,1,2, \ldots$ so as to get the expression of $Z(x, \lambda)$. Substituting (3.8) into (3.7) leads to

$$
\sum_{k=0}^{\infty} \frac{P_{-k}^{\prime}(x)}{\lambda^{k}} E(x, \lambda)+\sum_{k=0}^{\infty} \frac{P_{-k}(x)}{\lambda^{k}} E^{\prime}(x, \lambda)=\left[\lambda \widetilde{\Lambda}_{1}(x)+\widetilde{\Lambda}_{0}+\lambda^{-1} \widetilde{\Lambda}_{-1}\right] \sum_{k=0}^{\infty} \frac{P_{-k}(x)}{\lambda^{k}} E(x, \lambda) .
$$

Then each coefficient with the same power of $\lambda$ in both sides of above equation must be equal, i.e.,

$$
\begin{array}{rll}
\lambda & : \quad P_{0}(x) \widetilde{\Lambda}_{1}(x)=\widetilde{\Lambda}_{1}(x) P_{0}(x) \\
1 & : & P_{0}^{\prime}(x)+P_{-1}(x) \widetilde{\Lambda}_{1}(x)=\widetilde{\Lambda}_{1}(x) P_{-1}(x)+\widetilde{\Lambda}_{0} P_{0}(x), \\
\lambda^{-1}: & P_{-1}^{\prime}(x)+P_{-2}(x) \widetilde{\Lambda}_{1}(x)=\widetilde{\Lambda}_{1}(x) P_{-2}(x)+\widetilde{\Lambda}_{0} P_{-1}(x)+\widetilde{\Lambda}_{-1} P_{0}(x), \\
\lambda^{-k}: & P_{-k}^{\prime}(x)+P_{-(k+1)}(x) \widetilde{\Lambda}_{1}(x)=\widetilde{\Lambda}_{1}(x) P_{-(k+1)}(x)+\widetilde{\Lambda}_{0} P_{-k}(x)+\widetilde{\Lambda}_{-1} P_{-k+1}(x) .
\end{array}
$$

(3.10) implies that $P_{0}$ is a diagonal matrix. By (3.11), we have $P_{0}^{\prime}=\operatorname{diag} \widetilde{\Lambda}_{0} \cdot P_{0}(x)$. From the expression of $\widetilde{\Lambda}_{0}$, a direct calculation yields $P_{0}=I$.

Now let us calculate $P_{-1}$. Assume that

$$
\sqrt{\frac{I_{\rho}}{E I}} \neq \sqrt{\frac{\rho}{k}}
$$

Firstly, by (3.11), we get the expression of the non-diagonal entries of $P_{-1}=\left(P_{-1}^{i j}\right)_{4 \times 4}$ given as follows

$$
\left\{\begin{array}{l}
P_{-1}^{12}=\frac{\frac{\sqrt{I_{\rho} k}}{\sqrt{E I \rho}}}{2\left(\sqrt{\frac{I_{\rho}}{E I}}-\sqrt{\frac{\rho}{k}}\right)}, \quad P_{-1}^{13}=0, \quad P_{-1}^{14}=\frac{\frac{\sqrt{I_{\rho} k}}{\sqrt{E I \rho}}}{2\left(\sqrt{\frac{I_{\rho}}{E I}}+\sqrt{\frac{\rho}{k}}\right)}, \\
P_{-1}^{21}=\frac{\frac{\sqrt{\rho k}}{\sqrt{I_{\rho} E I}}}{2\left(\sqrt{\frac{I_{\rho}}{E I}}-\sqrt{\frac{\rho}{k}}\right)}, \quad P_{-1}^{23}=\frac{\frac{\sqrt{\rho k}}{\sqrt{I_{\rho} E I}}}{-2\left(\sqrt{\frac{I_{\rho}}{E I}}+\sqrt{\frac{\rho}{k}}\right)}, \quad P_{-1}^{24}=0 \\
P_{-1}^{31}=0, \quad P_{-1}^{32}=\frac{\frac{\sqrt{I \rho k}}{\sqrt{E I \rho}}}{-2\left(\sqrt{\frac{I_{\rho}}{E I}}+\sqrt{\frac{\rho}{k}}\right)}, \quad P_{-1}^{34}=\frac{\frac{\sqrt{I_{\rho} k}}{\sqrt{E I \rho}}}{-2\left(\sqrt{\frac{I_{\rho}}{E I}}-\sqrt{\frac{\rho}{k}}\right)}, \\
P_{-1}^{41}=\frac{\frac{\sqrt{\rho k}}{\sqrt{I_{\rho} E I}}}{2\left(\sqrt{\frac{I_{\rho}}{E I}}+\sqrt{\frac{\rho}{k}}\right)}, \quad P_{-1}^{42}=0, \quad P_{-1}^{43}=\frac{\frac{\sqrt{\rho k}}{\sqrt{I \rho E I}}}{-2\left(\sqrt{\frac{I_{\rho}}{E I}}-\sqrt{\frac{\rho}{k}}\right)}
\end{array}\right.
$$


Then, let us calculate $\operatorname{diag} P_{-1}$. From (3.12), we have

$$
\frac{\mathrm{d} P_{-1}^{i i}}{\mathrm{~d} x}=\widetilde{\Lambda}_{0}^{i i} P_{-1}^{i i}+\sum_{j=1, j \neq i}^{4} \widetilde{\Lambda}_{0}^{i j} P_{-1}^{j i}+\widetilde{\Lambda}_{-1}^{i i} P_{0}^{i i}
$$

which yields that the diagonal entries of $P_{-1}$ are

$$
\begin{cases}P_{-1}^{11}=x\left[\sum_{j=1, j \neq 1}^{4} \frac{\widetilde{\Lambda}_{0}^{1 j} \widetilde{\Lambda}_{0}^{j 1}}{\sqrt{\frac{\rho}{k}}-\widetilde{\Lambda}_{1}^{j j}}\right], \quad P_{-1}^{22}=x\left[\sum_{j=1, j \neq 2}^{4} \frac{\widetilde{\Lambda}_{0}^{2 j} \widetilde{\Lambda}_{0}^{j^{2}}}{\sqrt{\frac{I_{\rho}}{E I}}-\widetilde{\Lambda}_{1}^{j j}}+\frac{k}{2 \sqrt{I_{\rho} E I}}\right], \\ P_{-1}^{33}=x\left[\sum_{j=1, j \neq 3}^{4} \frac{\widetilde{\Lambda}_{0}^{3 j} \widetilde{\Lambda}_{0}^{j 3}}{-\sqrt{\frac{\rho}{k}}-\widetilde{\Lambda}_{1}^{j j}}\right], & P_{-1}^{44}=x\left[\sum_{j=1, j \neq 4}^{4} \frac{\widetilde{\Lambda}_{0}^{4 j} \tilde{\Lambda}_{0}^{j 4}}{-\sqrt{\frac{I_{\rho}}{E I}}-\widetilde{\Lambda}_{1}^{j j}}-\frac{k}{2 \sqrt{I_{\rho} E I}}\right] .\end{cases}
$$

Thus, all entries of $P_{-1}$ have been identified. Similarly, we can calculate $P_{-i}, i=2,3, \ldots$ by (3.13). Therefore, the following result holds.

Lemma 3.1. Suppose that condition (3.14) is fulfilled. Under the transformation $Y(x)=T_{0} Z(x)$, the expression of the asymptotic fundamental matrix solution to (3.6) is given as follows

$$
\widetilde{E}(x, \lambda)=\sum_{k=0}^{\infty} \frac{P_{-k}(x)}{\lambda^{k}} E(x, \lambda),
$$

where $E(x, \lambda)$ is defined by (3.9), $P_{0}=I, P_{-1}$ are given by (3.15) and (3.16) and $P_{-k, k=2,3, \ldots}$ can be calculated similarly. Thus, by the inverse transformation, the fundamental matrix solution to (3.4) is $T_{0} \widetilde{E}(x, \lambda)$.

\subsection{Asymptotic spectrum of $\mathcal{A}$}

In this subsection, we shall consider the distribution of the spectrum of $\mathcal{A}$ using the Birkhöff asymptotic technique (see [7]). According to (3.1), we have

$$
p(1)=p(0) \mathrm{e}^{-\tau_{1} \lambda}=\lambda w(1) \mathrm{e}^{-\tau_{1} \lambda}, \quad q(1)=q(0) \mathrm{e}^{-\tau_{2} \lambda}=\lambda \varphi(1) \mathrm{e}^{-\tau_{2} \lambda} .
$$

Substituting (3.18) into the last two boundary conditions of (3.1) yields

$$
k\left(w_{x}-\varphi\right)(1)=-\alpha_{1} \mu_{1} \lambda w(1)-\alpha_{1}\left(1-\mu_{1}\right) \lambda w(1) \mathrm{e}^{-\tau_{1} \lambda}, E I \varphi_{x}(1)=-\alpha_{2} \mu_{2} \lambda \varphi(1)-\alpha_{2}\left(1-\mu_{2}\right) \lambda \varphi(1) \mathrm{e}^{-\tau_{2} \lambda} .
$$

Let $Y$ be defined by (3.3). Together with (3.4), we translate (3.1) into the following matrix form

$$
\left\{\begin{array}{l}
\frac{\mathrm{d} Y}{\mathrm{~d} x}=\widetilde{A} Y \\
B_{1} Y(0)+B_{2} Y(1)=0
\end{array}\right.
$$

where $\widetilde{A}$ is given by (3.4) and

$$
B_{1}:=\left[\begin{array}{cccc}
1 & 0 & 0 & 0 \\
0 & 1 & 0 & 0 \\
0 & 0 & 0 & 0 \\
0 & 0 & 0 & 0
\end{array}\right], \quad B_{2}:=\left[\begin{array}{cccc}
0 & 0 & 0 & 0 \\
0 & 0 & 0 & 0 \\
\alpha_{1} \mu_{1}+\alpha_{1}\left(1-\mu_{1}\right) \mathrm{e}^{-\tau_{1} \lambda} & -\frac{k}{\lambda} & k & 0 \\
0 & \alpha_{2} \mu_{2}+\alpha_{2}\left(1-\mu_{2}\right) \mathrm{e}^{-\tau_{2} \lambda} & 0 & E I
\end{array}\right] .
$$

Set

$$
H(\lambda):=B_{1} T_{0}+B_{2} T_{0} \widetilde{E}(1, \lambda)
$$

where $T_{0}$ and $\widetilde{E}(1, \lambda)$ are given by $(3.5)$ and (3.17), respectively. 
Then, similarly with the proof in [23], we get the following result:

Lemma 3.2. Let $\mathcal{A}$ and $\mathcal{H}$ be defined as before. Then $\lambda \in \sigma(\mathcal{A})$ if and only if $\lambda$ satisfies

$$
\Delta(\lambda):=\operatorname{det} H(\lambda)=0 .
$$

Since all coefficients in $\Delta(\lambda)$ are real constants, we have:

Corollary 3.1. Let $\mathcal{A}$ and $\mathcal{H}$ be defined as before. Then the eigenvalues of $\mathcal{A}$ distribute in conjugate pairs on the complex plane, i.e., $\sigma(\mathcal{A})=\overline{\sigma(\mathcal{A})}$.

In order to get the spectrum of $\mathcal{A}$, from Lemma 3.2 , it is sufficient to identify the zeros of $\Delta(\lambda)$. For convenience, set

$$
[B]_{1}:=B+\mathcal{O}\left(\lambda^{-1}\right) .
$$

Then $\widetilde{E}(1, \lambda)$ can be rewritten as follows

$$
\widetilde{E}(1, \lambda)=\left[\begin{array}{llll}
{[1]_{1} \mathrm{e}^{\lambda \sqrt{\frac{\rho}{k}}}} & {[0]_{1} \mathrm{e}^{\lambda \sqrt{\frac{T_{\rho}}{E I}}}} & {[0]_{1} \mathrm{e}^{-\lambda \sqrt{\frac{\rho}{k}}}} & {[0]_{1} \mathrm{e}^{-\lambda \sqrt{\frac{T_{\rho}}{E I}}}} \\
{[0]_{1} \mathrm{e}^{\lambda \sqrt{\frac{\rho}{k}}}} & {[1]_{1} \mathrm{e}^{\lambda \sqrt{\frac{T_{\rho}}{E I}}}} & {[0]_{1} \mathrm{e}^{-\lambda \sqrt{\frac{\rho}{k}}}} & {[0]_{1} \mathrm{e}^{-\lambda \sqrt{\frac{T_{\rho}}{E I}}}} \\
{[0]_{1} \mathrm{e}^{\lambda \sqrt{\frac{\rho}{k}}}} & {[0]_{1} \mathrm{e}^{\lambda \sqrt{\frac{T_{\rho}}{E I}}}} & {[1]_{1} \mathrm{e}^{-\lambda \sqrt{\frac{\rho}{k}}}} & {[0]_{1} \mathrm{e}^{-\lambda \sqrt{\frac{I_{\rho}}{E I}}}} \\
{[0]_{1} \mathrm{e}^{\lambda \sqrt{\frac{\rho}{k}}}} & {[0]_{1} \mathrm{e}^{\lambda \sqrt{\frac{T_{\rho}}{E I}}}} & {[0]_{1} \mathrm{e}^{-\lambda \sqrt{\frac{\rho}{k}}}} & {[1]_{1} \mathrm{e}^{-\lambda \sqrt{\frac{T_{\rho}}{E I}}}}
\end{array}\right]
$$

Hence,

$$
\begin{aligned}
& H(\lambda)=B_{1} T_{0}+B_{2} T_{0} \widetilde{E}(1, \lambda) \\
& =\left[\begin{array}{ll}
{[1]_{1}+[0]_{1} \mathrm{e}^{\lambda \sqrt{\frac{\rho}{k}}}} & {[0]_{1} \mathrm{e}^{\lambda \sqrt{\frac{I_{\rho}}{E I}}}} \\
{[0]_{1} \mathrm{e}^{\lambda \sqrt{\frac{\rho}{k}}}} & {[1]_{1}+[0]_{1} \mathrm{e}^{\lambda \sqrt{\frac{I_{\rho}}{E I}}}} \\
{\left[\alpha_{1} \mu_{1}+\alpha_{1}\left(1-\mu_{1}\right) \mathrm{e}^{-\tau_{1} \lambda}+\sqrt{\rho k} \mathrm{e}_{1} \mathrm{e}^{\lambda \sqrt{\frac{\rho}{k}}}\right.} & -[0]_{1} \mathrm{e}^{\lambda \sqrt{\frac{T_{\rho}}{E I}}} \\
{[0]_{1} \mathrm{e}^{\lambda \sqrt{\frac{\rho}{k}}}} & {\left[\alpha_{2} \mu_{2}+\alpha_{2}\left(1-\mu_{2}\right) \mathrm{e}^{-\tau_{2} \lambda}+\sqrt{I_{\rho} E I}\right]_{1} \mathrm{e}^{\lambda \sqrt{\frac{T_{\rho}}{E I}}}}
\end{array}\right. \\
& \left.\begin{array}{ll}
{[1]_{1}+[0]_{1} \mathrm{e}^{-\lambda \sqrt{\frac{\rho}{k}}}} & {[0]_{1} \mathrm{e}^{-\lambda \sqrt{\frac{T_{\rho}}{E I}}}} \\
{[0]_{1} \mathrm{e}^{-\lambda \sqrt{\frac{\rho}{k}}}} & {[1]_{1}+[0]_{1} \mathrm{e}^{-\lambda \sqrt{\frac{T_{\rho}}{E I}}}} \\
{\left[\alpha_{1} \mu_{1}+\alpha_{1}\left(1-\mu_{1}\right) \mathrm{e}^{-\tau_{1} \lambda}-\sqrt{\rho k]_{1}} \mathrm{e}^{-\lambda \sqrt{\frac{\rho}{k}}}\right.} & -[0]_{1} \mathrm{e}^{-\lambda \sqrt{\frac{T_{\rho}}{E I}}} \\
{[0]_{1} \mathrm{e}^{-\lambda \sqrt{\frac{\rho}{k}}}} & {\left[\alpha_{2} \mu_{2}+\alpha_{2}\left(1-\mu_{2}\right) \mathrm{e}^{-\tau_{2} \lambda}-\sqrt{I_{\rho} E I}\right]_{1} \mathrm{e}^{-\lambda} \sqrt{\frac{\rho_{\rho}}{E I}}}
\end{array}\right]_{4 \times 4}
\end{aligned}
$$

Therefore, when $\Re \lambda \rightarrow+\infty$,

$$
\left|\lim _{\Re \lambda \rightarrow+\infty} \frac{\Delta(\lambda)}{\mathrm{e}^{\lambda\left(\sqrt{\frac{\rho}{k}}+\sqrt{\frac{I_{\rho}}{E I}}\right)}}\right|=\left(\alpha_{1} \mu_{1}+\sqrt{\rho k}\right)\left(\alpha_{2} \mu_{2}+\sqrt{I_{\rho} E I}\right) .
$$

Similarly, when $\Re \lambda \rightarrow-\infty$,

$$
\left|\lim _{\Re \lambda \rightarrow-\infty} \frac{\Delta(\lambda)}{\left.\mathrm{e}^{-\lambda\left(\sqrt{\frac{\rho}{k}}+\sqrt{\frac{\rho_{\rho}}{E I}}+\tau_{1}+\tau_{2}\right.}\right)}\right|=\alpha_{1} \alpha_{2}\left(1-\mu_{1}\right)\left(1-\mu_{2}\right) .
$$

Since $\mu_{i}<1, i=1,2,(3.21)$ and (3.22) together with the dissipativity of $\mathcal{A}$ imply the following result: 
Theorem 3.1. Let $\mathcal{A}$ and $\mathcal{H}$ be defined as before. Then the spectrum of $\mathcal{A}$ is contained in a strip parallel to imaginary axis, i.e., there exists a sufficiently large positive constant $h$ such that

$$
\sigma(\mathcal{A})=\{\lambda \in \mathbb{C} \mid \Delta(\lambda)=0\} \subset\{\lambda \in \mathbb{C} \mid-h \leq \Re \lambda \leq 0\} .
$$

Thus, when $|\Re \lambda| \leq h$ and $|\lambda|$ is large enough, we have

$$
\begin{aligned}
& \Delta(\lambda)=\operatorname{det}\left(B_{1}(0) T_{0}+B_{2}(1) T_{0} \tilde{E}(1, \lambda)\right) \\
& =\operatorname{det}\left[\begin{array}{ll}
{[1]_{1}+[0]_{1} \mathrm{e}^{\lambda \sqrt{\frac{\rho}{k}}}} & {[0]_{1} \mathrm{e}^{\lambda \sqrt{\frac{I_{\rho}}{E I}}}} \\
{[0]_{1} \mathrm{e}^{\lambda \sqrt{\frac{\rho}{k}}}} & {[1]_{1}+[0]_{1} \mathrm{e}^{\lambda \sqrt{\frac{T_{\rho}}{E I}}}} \\
{\left[\alpha_{1} \mu_{1}+\alpha_{1}\left(1-\mu_{1}\right) \mathrm{e}^{-\tau_{1} \lambda}+\sqrt{\rho k}\right]_{1} \mathrm{e}^{\lambda \sqrt{\frac{\rho}{k}}}} & -[0]_{1} \mathrm{e}^{\lambda \sqrt{\frac{I_{\rho}}{E T}}} \\
{[0]_{1} \mathrm{e}^{\lambda \sqrt{\frac{\rho}{k}}}} & {\left[\alpha_{2} \mu_{2}+\alpha_{2}\left(1-\mu_{2}\right) \mathrm{e}^{-\tau_{2} \lambda}+\sqrt{I_{\rho} E I}\right]_{1} \mathrm{e}^{\lambda \sqrt{\frac{I_{\rho}}{E I}}}}
\end{array}\right. \\
& {[1]_{1}+[0]_{1} \mathrm{e}^{-\lambda \sqrt{\frac{\rho}{k}}} \quad[0]_{1} \mathrm{e}^{-\lambda \sqrt{\frac{T_{\rho}}{E I}}}} \\
& {[0]_{1} \mathrm{e}^{-\lambda \sqrt{\frac{\rho}{k}}} \quad[1]_{1}+[0]_{1} \mathrm{e}^{-\lambda \sqrt{\frac{T_{\rho}}{E T}}}} \\
& {\left[\alpha_{1} \mu_{1}+\alpha_{1}\left(1-\mu_{1}\right) \mathrm{e}^{-\tau_{1} \lambda}-\sqrt{\rho \kappa}\right]_{1} \mathrm{e}^{-\lambda \sqrt{\frac{\rho}{k}}}-[0]_{1} \mathrm{e}^{-\lambda \sqrt{\frac{T_{\rho}}{E T}}}} \\
& \left.[0]_{1} \mathrm{e}^{-\lambda \sqrt{\frac{\rho}{k}}} \quad\left[\alpha_{2} \mu_{2}+\alpha_{2}\left(1-\mu_{2}\right) \mathrm{e}^{-\tau_{2} \lambda}-\sqrt{I_{\rho} E I}\right]_{1} \mathrm{e}^{-\lambda \sqrt{\frac{T_{\rho}}{E I}}}\right]_{4 \times 4} \\
& =\Delta_{1}(\lambda) \Delta_{2}(\lambda)+\mathcal{O}\left(\lambda^{-1}\right)
\end{aligned}
$$

where

and

$$
\Delta_{1}(\lambda):=\operatorname{det}\left[\begin{array}{c}
1 \\
{\left[\alpha_{1} \mu_{1}+\alpha_{1}\left(1-\mu_{1}\right) \mathrm{e}^{-\tau_{1} \lambda}+\sqrt{\rho k}\right] \mathrm{e}^{\lambda \sqrt{\frac{\rho}{k}}}} \\
{\left[\alpha_{1} \mu_{1}+\alpha_{1}\left(1-\mu_{1}\right) \mathrm{e}^{-\tau_{1} \lambda}-\sqrt{\rho k}\right] \mathrm{e}^{-\lambda \sqrt{\frac{e}{k}}}}
\end{array}\right]
$$

$$
\Delta_{2}(\lambda):=\operatorname{det}\left[\begin{array}{cc}
1 & 1 \\
{\left[\alpha_{2} \mu_{2}+\alpha_{2}\left(1-\mu_{2}\right) \mathrm{e}^{-\tau_{2} \lambda}+\sqrt{\left.I_{\rho} E I\right]} \mathrm{e}^{\lambda} \sqrt{\frac{I_{\rho}}{E I}}\right.} & {\left[\alpha_{2} \mu_{2}+\alpha_{2}\left(1-\mu_{2}\right) \mathrm{e}^{-\tau_{2} \lambda}-\sqrt{\left.I_{\rho} E I\right]} \mathrm{e}^{-\lambda \sqrt{\frac{I_{\rho}}{E I}}}\right.}
\end{array}\right] .
$$

According to the discussion above, we have:

Lemma 3.3. Let $\mathcal{A}$ and $\mathcal{H}$ be defined as before. Then the asymptotic values of the spectrum of $\mathcal{A}$ can be determined by $\Delta_{1}(\lambda)=0$ and $\Delta_{2}(\lambda)=0$ which are given by (3.23) and (3.24).

Hence, let us discuss the zeros of $\Delta_{1}(\lambda)$ and $\Delta_{2}(\lambda)$, which can imply the asymptotic distribution of the spectrum of $\mathcal{A}$.

Lemma 3.4. Let $\mathcal{A}$ and $\mathcal{H}$ be defined as before and the condition (3.14) be fulfilled. Then the zeros of $\Delta_{i}(\lambda)$, $i=1,2$ are at most of degree two and separated. Furthermore, if $\sqrt{\frac{\rho}{k}} / \tau_{1}$ and $\sqrt{\frac{I_{\rho}}{E I}} / \tau_{2}$ are irrational constants, then all of the zeros of $\Delta_{i}(\lambda), i=1,2$ are simple.

Proof. Firstly, we consider the zeros of $\Delta_{1}(\lambda)$. A direct calculation yields

$$
\Delta_{1}(\lambda)=-2 \alpha_{1} \mu_{1} \sinh \lambda \sqrt{\frac{\rho}{k}}-2 \sqrt{\rho k} \cosh \lambda \sqrt{\frac{\rho}{k}}-2\left(1-\mu_{1}\right) \alpha_{1} \mathrm{e}^{-\tau_{1} \lambda} \sinh \lambda \sqrt{\frac{\rho}{k}} .
$$

Then differentiating (3.25), we have

$$
\left\{\begin{aligned}
\Delta_{1}^{\prime}(\lambda)= & -2 \alpha_{1} \mu_{1} \sqrt{\frac{\rho}{k}} \cosh \lambda \sqrt{\frac{\rho}{k}}-2 \rho \sinh \lambda \sqrt{\frac{\rho}{k}}-2 \sqrt{\frac{\rho}{k}}\left(1-\mu_{1}\right) \alpha_{1} \mathrm{e}^{-\tau_{1} \lambda} \cosh \lambda \sqrt{\frac{\rho}{k}} \\
& +2\left(1-\mu_{1}\right) \alpha_{1} \tau_{1} \mathrm{e}^{-\tau_{1} \lambda} \sinh \lambda \sqrt{\frac{\rho}{k}} \\
\Delta_{1}^{\prime \prime}(\lambda)= & -2 \alpha_{1} \mu_{1} \frac{\rho}{k} \sinh \lambda \sqrt{\frac{\rho}{k}}-2 \frac{\rho}{k} \sqrt{\rho k} \cosh \lambda \sqrt{\frac{\rho}{k}}-2\left(1-\mu_{1}\right) \alpha_{1} \frac{\rho}{k} \mathrm{e}^{-\tau_{1} \lambda} \sinh \lambda \sqrt{\frac{\rho}{k}} \\
& +4 \sqrt{\frac{\rho}{k}}\left(1-\mu_{1}\right) \alpha_{1} \tau_{1} \mathrm{e}^{-\tau_{1} \lambda} \cosh \lambda \sqrt{\frac{\rho}{k}}-2\left(1-\mu_{1}\right) \alpha_{1} \tau_{1}^{2} \mathrm{e}^{-\tau_{1} \lambda} \sinh \lambda \sqrt{\frac{\rho}{k}}
\end{aligned}\right.
$$


We shall show that the zeros of $\Delta_{1}(\lambda)$ are at most of degree two. It only needs to prove that for any $\tilde{\lambda} \in \mathbb{C}$, if $\Delta_{1}(\widetilde{\lambda})=\Delta_{1}^{\prime}(\underset{\widetilde{\lambda}}{\widetilde{\lambda}})=0$, it must hold that $\Delta_{1}^{\prime \prime}(\widetilde{\lambda}) \neq 0$.

Since $\Delta_{1}(\widetilde{\lambda})=0$ implies $\sinh \widetilde{\lambda} \sqrt{\frac{\rho}{k}} \neq 0$, we have

$$
\begin{gathered}
\frac{\Delta_{1}(\widetilde{\lambda})}{\sinh \widetilde{\lambda} \sqrt{\frac{\rho}{k}}}=-2 \alpha_{1} \mu_{1}-2 \sqrt{\rho k} \frac{\cosh \widetilde{\lambda} \sqrt{\frac{\rho}{k}}}{\sinh \widetilde{\lambda} \sqrt{\frac{\rho}{k}}}-2\left(1-\mu_{1}\right) \alpha_{1} \mathrm{e}^{-\tau_{1} \widetilde{\lambda}} \\
\frac{\Delta_{1}^{\prime}(\widetilde{\lambda})}{\sinh \widetilde{\lambda} \sqrt{\frac{\rho}{k}}}=-2 \alpha_{1} \mu_{1} \sqrt{\frac{\rho}{k}} \frac{\cosh \widetilde{\lambda} \sqrt{\frac{\rho}{k}}}{\sinh \widetilde{\lambda} \sqrt{\frac{\rho}{k}}}-2 \rho-2\left(1-\mu_{1}\right) \alpha_{1} \sqrt{\frac{\rho}{k}} \mathrm{e}^{-\tau_{1} \widetilde{\lambda}} \frac{\cosh \widetilde{\lambda} \sqrt{\frac{\rho}{k}}}{\sinh \widetilde{\lambda} \sqrt{\frac{\rho}{k}}}+2\left(1-\mu_{1}\right) \alpha_{1} \tau_{1} \mathrm{e}^{-\tau_{1} \widetilde{\lambda}} .
\end{gathered}
$$

Hence, $\Delta_{1}(\widetilde{\lambda})=0$ yields

$$
\left(1-\mu_{1}\right) \alpha_{1} \mathrm{e}^{-\tau_{1} \widetilde{\lambda}}=-\alpha_{1} \mu_{1}-\sqrt{\rho k} \frac{\cosh \widetilde{\lambda} \sqrt{\frac{\rho}{k}}}{\sinh \widetilde{\lambda} \sqrt{\frac{\rho}{k}}}
$$

Then, substituting (3.29) into (3.28) leads to

$$
\frac{\Delta_{1}^{\prime}(\widetilde{\lambda})}{\sinh \widetilde{\lambda} \sqrt{\frac{\rho}{k}}}=2 \rho\left(\frac{\cosh \tilde{\lambda} \sqrt{\frac{\rho}{k}}}{\sinh \widetilde{\lambda} \sqrt{\frac{\rho}{k}}}\right)^{2}-2 \tau_{1} \sqrt{\rho k} \frac{\cosh \tilde{\lambda} \sqrt{\frac{\rho}{k}}}{\sinh \widetilde{\lambda} \sqrt{\frac{\rho}{k}}}-2 \rho-2 \tau_{1} \alpha_{1} \mu_{1} .
$$

Thus, from $\Delta_{1}^{\prime}(\widetilde{\lambda})=0$, it holds that

$$
\frac{\cosh \widetilde{\lambda} \sqrt{\frac{\rho}{k}}}{\sinh \widetilde{\lambda} \sqrt{\frac{\rho}{k}}}=\frac{\tau_{1} \sqrt{\rho k} \pm \sqrt{\tau_{1}^{2} \rho k+4 \rho\left(\rho+\tau_{1} \alpha_{1} \mu_{1}\right)}}{2 \rho} .
$$

According to (3.26), we have

$$
\begin{aligned}
\frac{\Delta_{1}^{\prime \prime}(\tilde{\lambda})}{\sinh \widetilde{\lambda} \sqrt{\frac{\rho}{k}}} & =\frac{\rho}{k} \frac{\Delta_{1}(\tilde{\lambda})}{\sinh \widetilde{\lambda} \sqrt{\frac{\rho}{k}}}+4\left(1-\mu_{1}\right) \alpha_{1} \tau_{1} \mathrm{e}^{-\tau_{1} \tilde{\lambda}} \sqrt{\frac{\rho}{k}} \frac{\cosh \widetilde{\lambda} \sqrt{\frac{\rho}{k}}}{\sinh \widetilde{\lambda} \sqrt{\frac{\rho}{k}}}-2\left(1-\mu_{1}\right) \alpha_{1} \tau_{1}^{2} \mathrm{e}^{-\tau_{1} \tilde{\lambda}} \\
& =2\left(1-\mu_{1}\right) \alpha_{1} \tau_{1} \mathrm{e}^{-\tau_{1} \tilde{\lambda}}\left[2 \sqrt{\frac{\rho}{k}} \frac{\cosh \widetilde{\lambda} \sqrt{\frac{\rho}{k}}}{\sinh \widetilde{\lambda} \sqrt{\frac{\rho}{k}}}-\tau_{1}\right] .
\end{aligned}
$$

Therefore, (3.30) leads to $\frac{\Delta_{1}^{\prime \prime}(\widetilde{\lambda})}{\sinh \tilde{\lambda} \sqrt{\frac{\rho}{k}}} \neq 0$, which implies that the zeros of $\Delta_{1}(\lambda)$ are at most of degree two.

Now, we suppose that $\tilde{\lambda}$ is a zero of $\Delta_{1}(\lambda)$ of degree two. Then (3.29) and (3.30) must hold.

From (3.30), a direct calculation yields

$$
\mathrm{e}^{2 \tilde{\lambda} \sqrt{\frac{\rho}{k}}}= \begin{cases}\frac{-\rho-\frac{1}{2}\left(\tau_{1} \sqrt{\rho k}+\sqrt{\tau_{1}^{2} \rho k+4 \rho\left(\rho+\tau_{1} \alpha_{1} \mu_{1}\right)}\right)}{\rho-\frac{1}{2}\left(\tau_{1} \sqrt{\rho k}+\sqrt{\tau_{1}^{2} \rho k+4 \rho\left(\rho+\tau_{1} \alpha_{1} \mu_{1}\right)}\right)}, & \text { take }+\operatorname{sign} \text { in }(3.30), \\ \frac{-\rho-\frac{1}{2}\left(\tau_{1} \sqrt{\rho k}-\sqrt{\tau_{1}^{2} \rho k+4 \rho\left(\rho+\tau_{1} \alpha_{1} \mu_{1}\right)}\right)}{\rho-\frac{1}{2}\left(\tau_{1} \sqrt{\rho k}-\sqrt{\tau_{1}^{2} \rho k+4 \rho\left(\rho+\tau_{1} \alpha_{1} \mu_{1}\right)}\right)}, & \text { take }-\operatorname{sign} \text { in (3.30). }\end{cases}
$$

Substituting (3.30) into (3.29), we get

$$
\mathrm{e}^{\tau_{1} \tilde{\lambda}}= \begin{cases}\frac{2 \rho\left(1-\mu_{1}\right) \alpha_{1}}{-2 \alpha_{1} \mu_{1} \rho-\sqrt{\rho k}\left(\tau_{1} \sqrt{\rho k}+\sqrt{\tau_{1}^{2} \rho k+4 \rho\left(\rho+\tau_{1} \alpha_{1} \mu_{1}\right)}\right)}, & \text { take }+ \text { sign in }(3.30), \\ \frac{2 \rho\left(1-\mu_{1}\right) \alpha_{1}}{-2 \alpha_{1} \mu_{1} \rho-\sqrt{\rho k}\left(\tau_{1} \sqrt{\rho k}-\sqrt{\tau_{1}^{2} \rho k+4 \rho\left(\rho+\tau_{1} \alpha_{1} \mu_{1}\right)}\right)}, & \text { take }- \text { sign in }(3.30) .\end{cases}
$$


Set $\widetilde{\lambda}=x+y i, x, y \in \mathbb{R}$. Then by (3.31) and (3.32), we get that $\mathrm{e}^{\tau_{1} \tilde{\lambda}}, \mathrm{e}^{2 \tilde{\lambda} \sqrt{\frac{\rho}{k}}} \in \mathbb{R}$. Thus, $\sin 2 y \sqrt{\frac{\rho}{k}}=\sin y \tau_{1}=0$, which leads to

$$
2 y \sqrt{\frac{\rho}{k}}=n \pi, \quad \tau_{1} y=m \pi
$$

for some integers $n$ and $m$. Hence $\sqrt{\frac{\rho}{k}} / \tau_{1}=\frac{n}{2 m}$ is a rational number. Therefore, if $\sqrt{\frac{\rho}{k}} / \tau_{1}$ is an irrational constant, then all zeros of $\Delta_{1}(\lambda)$ are simple.

Let us now show the separability of the zeros of $\Delta_{1}(\lambda)$, i.e., there is a positive constant $\delta>0$ such that

$$
\inf _{\tilde{\xi} \neq \tilde{\lambda}, \tilde{\xi}, \tilde{\lambda} \in\left\{\lambda \mid \Delta_{1}(\lambda)=0\right\}}|\lambda-\xi| \geq \delta .
$$

We shall show the separability by the following two cases:

Case 1: $\sqrt{\frac{\rho}{k}} / \tau_{1}=\frac{n}{2 m}$ is rational with some integers $n, m$.

Set $z=\mathrm{e}^{\frac{\lambda \sqrt{\frac{\rho}{k}}}{n}}$. Then $\Delta_{1}(\lambda)=0$ is equivalent to the following equation:

$$
\left(\alpha_{1} \mu_{1}+\sqrt{\rho k}\right) z^{2 m+2 n}+\left(1-\mu_{1}\right) \alpha_{1} z^{2 n}-\left(\alpha_{1} \mu_{1}+\sqrt{\rho k}\right) z^{2 m}-\left(1-\mu_{1}\right) \alpha_{1}=0 .
$$

Since the number of the zeros of the above polynomial equation is at most $(2 m+2 n)$ and these zeros are separated, the zeros of $\Delta_{1}(\lambda)$ are also separated.

Case 2: $\sqrt{\frac{\rho}{k}} / \tau_{1}$ is irrational.

From (3.25), we can get that $\widetilde{\lambda} \in\left\{\lambda \mid \Delta_{1}(\lambda)=0\right\}$ implies

$$
0<\inf _{\tilde{\lambda} \in\left\{\lambda \mid \Delta_{1}(\lambda)=0\right\}}\left|\sinh \tilde{\lambda} \sqrt{\frac{\rho}{k}}\right| \leq \sup _{\tilde{\lambda} \in\left\{\lambda \mid \Delta_{1}(\lambda)=0\right\}}\left|\sinh \tilde{\lambda} \sqrt{\frac{\rho}{k}}\right|<\infty .
$$

(3.33) together with (3.31) and (3.32) yields that when $\sqrt{\frac{\rho}{k}} / \tau_{1}$ is irrational, $\inf _{\tilde{\lambda} \in\left\{\lambda \mid \Delta_{1}(\lambda)=0\right\}}\left|\Delta_{1}^{\prime}(\lambda)\right| \neq 0$, which yields that the zeros of $\Delta_{1}(\lambda)$ are separated by [20].

A similar method can be used to deal with the zeros of $\Delta_{2}(\lambda)$ and the corresponding result follows. The proof is complete.

Applying the Rouché Theorem (see [1]), we have the following result about the asymptotic spectrum of $\mathcal{A}$ :

Theorem 3.2. Let $\mathcal{A}$ and $\mathcal{H}$ be defined as before and the condition (3.14) be fulfilled. Then the asymptotic eigenvalues of $\mathcal{A}$ are at most of degree two and separated. Furthermore, if $\sqrt{\frac{\rho}{k}} / \tau_{1}$ and $\sqrt{\frac{I_{\rho}}{E I}} / \tau_{2}$ are irrational constants, then the asymptotic eigenvalues of $\mathcal{A}$ are simple.

\section{Riesz Basis PROperty of the (GENERALized) EIGENVECTORS}

In this section we shall discuss the Riesz basis property of the eigenvectors and generalized eigenvectors of $\mathcal{A}$. Firstly, let us establish the completeness of the eigenvectors and generalized eigenvectors of $\mathcal{A}$.

To this end, we define an auxiliary operator $\mathcal{A}_{0}$ in $\mathcal{H}$ :

$$
\mathcal{A}_{0}(w, z, p, \varphi, \psi, q)^{\tau}=\left(\begin{array}{c}
z \\
\rho^{-1} k w_{x x} \\
-\tau_{1}^{-1} p_{x} \\
\psi \\
I_{\rho}^{-1} E I \varphi_{x x} \\
-\tau_{2}^{-1} q_{x}
\end{array}\right)
$$


with domain

$$
\mathcal{D}\left(\mathcal{A}_{0}\right)=\left\{\begin{array}{l}
(w, z, p, \varphi, \psi, q)^{\tau} \in V^{2}(0,1) \times V^{1}(0,1) \times H^{1}(0,1) \times V^{2}(0,1) \times V^{1}(0,1) \times H^{1}(0,1) \\
\begin{array}{l}
k w^{\prime}(1)=-\alpha_{1} \mu_{1} z(1)-\alpha_{1}\left(1-\mu_{1}\right) p(1), z(1)=p(0) \\
E I \varphi^{\prime}(1)=-\alpha_{2} \mu_{2} \psi(1)-\alpha_{2}\left(1-\mu_{2}\right) q(1), v(1)=q(0)
\end{array}
\end{array}\right\} .
$$

Note that there is no coupled term of $(w, z, p)^{\tau}$ and $(\varphi, \psi, q)^{\tau}$ in $\mathcal{A}_{0}$ and $\mathcal{D}\left(\mathcal{A}_{0}\right)$. Thus, it becomes two wave systems, i.e.,

$$
\mathcal{A}_{0}^{1}(w, z, p)^{\tau}=\left(\begin{array}{c}
z \\
\rho^{-1} k w_{x x} \\
-\tau_{1}^{-1} p_{x}
\end{array}\right), \quad \quad \mathcal{A}_{0}^{2}(\varphi, \psi, q)^{\tau}=\left(\begin{array}{c}
\psi \\
I_{\rho}^{-1} E I \varphi_{x x} \\
-\tau_{2}^{-1} q_{x}
\end{array}\right)
$$

where

$$
\mathcal{D}\left(\mathcal{A}_{0}^{1}\right)=\left\{(w, z, p)^{\tau} \in V^{2}(0,1) \times V^{1}(0,1) \times H^{1}(0,1) \mid k w^{\prime}(1)=-\alpha_{1} \mu_{1} z(1)-\alpha_{1}\left(1-\mu_{1}\right) p(1), z(1)=p(0)\right\}
$$

and

$$
\mathcal{D}\left(\mathcal{A}_{0}^{2}\right)=\left\{(\varphi, \psi, q)^{\tau} \in V^{2}(0,1) \times V^{1}(0,1) \times H^{1}(0,1) \mid E I \varphi^{\prime}(1)=-\alpha_{2} \mu_{2} \psi(1)-\alpha_{2}\left(1-\mu_{2}\right) q(1), v(1)=q(0)\right\} .
$$

From Xu et al. [24], we get the following lemma:

Lemma 4.1. Let $\mathcal{A}_{0}^{1}$ and $\mathcal{A}_{0}^{2}$ be defined by (4.2). Then $\mathcal{A}_{0}^{1}$ and $\mathcal{A}_{0}^{2}$ generate a $C_{0}$ group on $V^{1}[0,1] \times L^{2}[0,1] \times$ $L^{2}[0,1]$, respectively.

Therefore, we have:

Lemma 4.2. Let $\mathcal{H}$ and $\mathcal{A}_{0}$ be defined by (2.1) and (4.1). Then $\mathcal{A}_{0}$ generates a $C_{0}$ group on $\mathcal{H}$.

Now, we shall use the property of $\mathcal{A}_{0}$ to discuss the corresponding property of $\mathcal{A}$. The following result holds.

Theorem 4.1. Let $\mathcal{A}$ and $\mathcal{H}$ be defined as before. Then $\mathcal{A}$ generates a $C_{0}$ group on $\mathcal{H}$. Furthermore, the system of the eigenvectors and generalized eigenvectors of $\mathcal{A}$ is complete in $\mathcal{H}$.

Proof. We define the transform operator $\mathcal{P}: \mathcal{H} \rightarrow \mathcal{H}$ as follows:

$$
\mathcal{P}(\hat{w}, z, p, \varphi, \psi, q)^{\tau}=(w, z, p, \varphi, \psi, q)^{\tau},
$$

where $w(x)=\hat{w}(x)+x \varphi(1)$. Then

$$
w_{x}=\hat{w}_{x}+\varphi(1) .
$$

Hence, the operator $\mathcal{P}$ maps $\mathcal{D}\left(\mathcal{A}_{0}\right)$ onto $\mathcal{D}(\mathcal{A})$. Obviously, $\mathcal{P}$ is a bijective operator. Then, for $(\hat{w}, z, p, \varphi, \psi, q)^{\tau} \in$ $\mathcal{D}\left(\mathcal{A}_{0}\right)$, we have

$$
\mathcal{P}^{-1} \mathcal{A} \mathcal{P}\left(\begin{array}{c}
\hat{w} \\
z \\
p \\
\varphi \\
\psi \\
q
\end{array}\right)=\left(\begin{array}{c}
z-x \varphi(1) \\
\rho^{-1} k\left(\hat{w}_{x x}-\varphi_{x}\right) \\
-\tau_{1}^{-1} p_{x} \\
\psi \\
\mathcal{I}_{\rho}^{-1} E I \varphi_{x x}+\mathcal{I}_{\rho}^{-1} k\left(\hat{w}_{x}+\varphi(1)-\varphi\right) \\
-\tau_{2}^{-1} q_{x}
\end{array}\right)
$$


Define the operator $\mathcal{B}$ on $\mathcal{H}$ as follows:

$$
\mathcal{B}\left(\begin{array}{c}
\hat{w} \\
z \\
p \\
\varphi \\
\psi \\
q
\end{array}\right)=\left(\begin{array}{c}
-x \varphi(1) \\
-\rho^{-1} k \varphi_{x} \\
0 \\
0 \\
\mathcal{I}_{\rho}^{-1} k\left(\hat{w}_{x}+\varphi(1)-\varphi\right) \\
0
\end{array}\right) .
$$

Then,

$$
\mathcal{A}_{0}+\mathcal{B}=\mathcal{P}^{-1} \mathcal{A P}
$$

A direction calculation yields that there exists a positive constant $\widehat{M}$, such that

$$
\left\|B(\hat{w}, z, p, \varphi, \psi, q)^{\tau}\right\| \leq \widehat{M}\left\|(\hat{w}, z, p, \varphi, \psi, q)^{\tau}\right\|
$$

which implies that $\mathcal{B}$ is a bounded linear operator. Transforming (4.5), we get

$$
\mathcal{A}=\mathcal{P} \mathcal{A}_{0} \mathcal{P}^{-1}+\mathcal{P B} \mathcal{P}^{-1}
$$

Thus, $\mathcal{A}$ is a bounded perturbation of $\mathcal{P} \mathcal{A}_{0} \mathcal{P}^{-1}$. We know from Lemma 4.2 that $\mathcal{A}_{0}$ generates a $C_{0}$ group on $\mathcal{H}$. Therefore, it holds that $\mathcal{A}$ also generates a $C_{0}$ group on $\mathcal{H}$ due to perturbation theorem.

Now let us show the completeness of the eigenvectors and generalized eigenvectors of $\mathcal{A}$. We assume that $\sigma(\mathcal{A})=\left\{\lambda_{k}, k \in \mathbb{N}\right\}$ due to Corollary 2.1. The completeness of the eigenvectors and generalized eigenvectors of $\mathcal{A}$ is that

$$
\operatorname{Span}(\mathcal{A})=\overline{\left\{\sum_{k} y_{k}, y_{k} \in E\left(\lambda_{k}, \mathcal{A}\right) \mathcal{H}, \lambda_{k} \in \sigma(\mathcal{A})\right\}}=\mathcal{H},
$$

where $E\left(\lambda_{k}, \mathcal{A}\right)$ is the Riesz projection corresponding to $\lambda_{k}$.

Assume that $Y_{0}=\left(y_{1}, y_{2}, y_{3}, y_{4}, y_{5}, y_{6}\right)^{\tau} \in \mathcal{H}, Y_{0} \perp \operatorname{Span}(\mathcal{A})$. Then for $\lambda \in \mathbb{C}, R^{*}(\lambda, \mathcal{A}) Y_{0}$ is a $\mathcal{H}$-valued entire function. Thus for any $F=\left(f^{1}, f^{2}, f^{3}, f^{4}, f^{5}, f^{6}\right)^{\tau} \in \mathcal{H}$, the function

$$
G(\lambda)=\left(F, R^{*}(\lambda, \mathcal{A}) Y_{0}\right)_{\mathcal{H}}, \quad \forall \lambda \in \mathbb{C}
$$

is an entire function. Since $\mathcal{A}$ generates a $C_{0}$ group, we have that $\lim _{\Re \lambda \rightarrow \infty} G(\lambda)=0$. In particular, for $\lambda \in \rho(\mathcal{A})$, it holds that $G(\lambda)=\left(R(\lambda, \mathcal{A}) F, Y_{0}\right)_{\mathcal{H}}$. Then the dissipativity of $\mathcal{A}$ ensures that $|G(\lambda)|$ is bounded on the domain $\Re \lambda \geq \widehat{\alpha}>0$. Since $G(\lambda)$ is an entire function of finite exponential type, the Phragmén-Lindelöf Theorem (see [25]) asserts that $|G(\lambda)|$ is bounded on the sectors region: $\widehat{\Omega}_{1}=\{\lambda \in \mathbb{C} \mid \Re \lambda \leq \widehat{\alpha}, \Im \lambda \geq 0\}$ and $\widehat{\Omega}_{2}=\{\lambda \in \mathbb{C} \mid \Im \lambda \leq 0, \Re \lambda \leq \widehat{\alpha}\}$ due to $G(\lambda)$ is bounded on their boundary lines $\Re \lambda=\widehat{\alpha}$ and $\Im \lambda=0$. Therefore, $|G(\lambda)|$ is uniformly bounded on $\mathbb{C}$, i.e., $|G(\lambda)| \leq M, \forall \lambda \in \mathbb{C}$.

Then by the Liouville's theorem, we have $G(\lambda) \equiv 0$ since $\lim _{\Re \lambda \rightarrow \infty} G(\lambda)=0$. Note that $G(\lambda)=\left(F, R^{*}(\lambda, \mathcal{A}) Y_{0}\right)_{\mathcal{H}}$ holds for any $F \in \mathcal{H}$. It must be $R^{*}(\lambda, \mathcal{A}) Y_{0}=0$, which means $Y_{0}=0$. Therefore, $\operatorname{Span}(\mathcal{A})=\mathcal{H}$. The desired result follows.

In order to get the Riesz basis property of the (generalized) eigenvectors, we need the following result from [22].

Theorem 4.2. Let $\mathcal{H}$ be a separable Hilbert space, and $\mathcal{A}$ be the generator of a $C_{0}$ semigroup $T(t)$ on $\mathcal{H}$. Suppose that:

(1) $\sigma(\mathcal{A})=\sigma_{1}(\mathcal{A}) \cup \sigma_{2}(\mathcal{A})$, where $\sigma_{2}(\mathcal{A})=\left\{\lambda_{k}\right\}_{k=1}^{\infty}$ consists of isolated eigenvalues of $\mathcal{A}$ with finite multiplicity;

(2) $\sup _{k>1} m_{a}\left(\lambda_{k}\right)<\infty$, where $m_{a}\left(\lambda_{k}\right)=\operatorname{dim} E\left(\lambda_{k}, \mathcal{A}\right) \mathcal{H}$ and $E\left(\lambda_{k}, \mathcal{A}\right)$ is the Riesz projector associated with $\lambda_{k}$;

(3) There is a constant $\alpha$ such that $\sup \left\{\operatorname{Re} \lambda \mid \lambda \in \sigma_{1}(\mathcal{A})\right\} \leq \alpha \leq \inf \left\{\operatorname{Re} \lambda \mid \lambda \in \sigma_{2}(\mathcal{A})\right\}$ and $\inf _{n \neq m}\left|\lambda_{n}-\lambda_{m}\right|>0$. 
Then the following assertions are true.

(i) There exist two $T(t)$-invariant closed subspaces $\mathcal{H}_{1}, \mathcal{H}_{2}$ with the property that $\sigma\left(\left.\mathcal{A}\right|_{\mathcal{H}_{1}}\right)=\sigma_{1}(\mathcal{A}), \sigma\left(\left.\mathcal{A}\right|_{\mathcal{H}_{2}}\right)=$ $\sigma_{2}(\mathcal{A}), E\left(\lambda_{k}, \mathcal{A}\right) \mathcal{H}_{2}$ forms a subspace Riesz basis for $\mathcal{H}_{2}$, and $\mathcal{H}=\overline{\mathcal{H}_{1} \oplus \mathcal{H}_{2}}$.

(ii) If $\sup _{k \geq 1}\left\|E\left(\lambda_{k}, \mathcal{A}\right)\right\|<\infty$, then $\mathcal{D}(\mathcal{A}) \subset \mathcal{H}_{1} \oplus \mathcal{H}_{2} \subset \mathcal{H}$.

(iii) $\mathcal{H}$ has the decomposition $\mathcal{H}=\mathcal{H}_{1} \oplus \mathcal{H}_{2}$ (topological direct sum) if and only if

$$
\sup _{n \geq 1}\left\|\sum_{k=1}^{n} E\left(\lambda_{k}, \mathcal{A}\right)\right\|<\infty .
$$

Applying Corollary 2.1, and Theorems 3.1, 3.2, 4.1, 4.2 to our problem, we get the following result:

Theorem 4.3. Let $\mathcal{H}$ and $\mathcal{A}$ be defined as before and the condition (2.8) be fulfilled. If (3.14) is satisfied, then there is a sequence of the eigenvectors and generalized eigenvectors of $\mathcal{A}$ which forms a Riesz basis for $\mathcal{H}$.

Proof. Set $\sigma_{1}(\mathcal{A})=\emptyset, \sigma_{2}(\mathcal{A})=\sigma(\mathcal{A})$. Theorems 3.1, 3.2 and Corollary 2.1 show that all hypotheses in Theorem 4.2 are fulfilled. So the results of Theorem 4.2 are true. Hence, there is a sequence of the eigenvectors and generalized eigenvectors of $\mathcal{A}$ that forms a subspace Riesz basis for $\mathcal{H}_{2}$. Theorem 4.1 says that the (generalized) eigenvectors is complete in $\mathcal{H}$, i.e., $\mathcal{H}_{2}=\mathcal{H}$. Therefore, the sequence is also a Riesz basis for $\mathcal{H}$. The proof is complete.

The Riesz basis property together with the uniform boundedness of multiplicities of the eigenvalues of $\mathcal{A}$ implies that:

Corollary 4.1. Under the conditions (2.8) and (3.14), the closed loop system (1.7) satisfies the spectrum determined growth condition, i.e., $\omega(\mathcal{A})=S(\mathcal{A})$, where $\omega(\mathcal{A})=\lim _{t \rightarrow \infty} \frac{1}{t} \ln \left\|\mathrm{e}^{\mathcal{A} t}\right\|$ is the growth order of $\mathrm{e}^{\mathcal{A} t}$ and $S(\mathcal{A})=\sup \{\Re \lambda \mid \lambda \in \sigma(\mathcal{A})\}$ is the spectral bound of $\mathcal{A}$.

\section{Stability of The System}

In this section, the stability of the closed loop system (1.7) is discussed. Under the choice of $\mu_{i}, i=1,2$, the asymptotic stability and exponential stability of the system (1.7) are gotten under certain conditions.

When $\mu_{1}<\frac{1}{2}$ or $\mu_{2}<\frac{1}{2}$, the following result holds.

Theorem 5.1. Let $\mathcal{A}$ and $\mathcal{H}$ be defined as before. When $\mu_{1}<\frac{1}{2}$ or $\mu_{2}<\frac{1}{2}$, the system (1.7) is always unstable.

Proof. We shall show that there always exists $\lambda \in \sigma(\mathcal{A})$ such that $\Re \lambda>0$ when $\mu_{1}<\frac{1}{2}$ or $\mu_{2}<\frac{1}{2}$, which leads to the instability of the system (1.7).

Let us consider the case $\mu_{1}<\frac{1}{2}$. Set $\widehat{y}:=\frac{\tau_{1}}{\sqrt{\frac{p}{k}}}$. If $\widehat{y}=\frac{2(2 m+1)}{2 n+1}, n, m \in \mathbb{N}$, we set

$$
\lambda:=\frac{1}{\sqrt{\frac{\rho}{k}}}\left[\sqrt{\frac{k}{\rho}} \widehat{\eta}+i\left(n+\frac{1}{2}\right) \pi+i 2 s(2 n+1) \pi\right], s \in \mathbb{Z}
$$

with some parameter $\widehat{\eta}$. Then a direct calculation yields

$$
2 \sqrt{\frac{\rho}{k}} \lambda=2 \widehat{\eta}+i(2 n+1) \pi+i 4 s(2 n+1) \pi, \lambda \tau_{1}=\frac{\lambda \sqrt{\frac{\rho}{k}}}{\sqrt{\frac{\rho}{k}}} \tau_{1}=\widehat{\eta} \tau_{1}+i(2 m+1) \pi+i 4 s(2 m+1) \pi, s \in \mathbb{Z} .
$$


Hence,

$$
\begin{aligned}
\Delta_{1}^{0}(\widehat{\eta}) & :=\mathrm{e}^{-\lambda \sqrt{\frac{\rho}{k}}} \Delta_{1}(\lambda) \\
& =\mathrm{e}^{-\lambda \sqrt{\frac{\rho}{k}}}\left[-2 \alpha_{1} \mu_{1} \sinh \lambda \sqrt{\frac{\rho}{k}}-2 \sqrt{\rho k} \cosh \lambda \sqrt{\frac{\rho}{k}}-2\left(1-\mu_{1}\right) \alpha_{1} \mathrm{e}^{-\tau_{1} \lambda} \sinh \lambda \sqrt{\frac{\rho}{k}}\right] \\
& =-\sqrt{\rho k}\left(1-\mathrm{e}^{-2 \widehat{\eta}}\right)-\left(\alpha_{1} \mu_{1}-\alpha_{1}\left(1-\mu_{1}\right) \mathrm{e}^{-\sqrt{\frac{k}{\rho}} \hat{\eta} \tau_{1}}\right)\left(1+\mathrm{e}^{-2 \widehat{\eta}}\right)
\end{aligned}
$$

where $\Delta_{1}(\lambda)$ is given by (3.23). Since $\Delta_{1}^{0}(0)=-2\left(2 \alpha_{1} \mu_{1}-\alpha_{1}\right)>0$ and $\lim _{\hat{\eta} \rightarrow+\infty} \Delta_{1}^{0}(\widehat{\eta})=-\sqrt{\rho k}-\alpha_{1} \mu_{1}<0$, there is at least one $\widehat{\eta}>0$ such that $\Delta_{1}^{0}(\widehat{\eta})=0$. Thus, for this $\widehat{\eta}$, the complex values $\lambda$ given by $(5.1)$ are the zeros of $\Delta_{1}(\lambda)$.

Now let $\widehat{y}$ be any positive real value. We can choose a sequence of rational numbers, $\widehat{y}_{n, m}:=\frac{2(2 m+1)}{2 n+1}$, such that $\lim _{n, m \rightarrow \infty} \widehat{y}_{n, m}=\widehat{y}$. Set $\widehat{y}=\widehat{y}_{n, m}+\varepsilon_{n, m}$, in which $\varepsilon_{n, m} \rightarrow 0$ when $n, m \rightarrow \infty$. We compare

$$
\Delta_{1}^{\widehat{y}}(\lambda):=\frac{1}{\lambda}\left[-2 \alpha_{1} \mu_{1} \sinh \lambda \sqrt{\frac{\rho}{k}}-2 \sqrt{\rho k} \cosh \lambda \sqrt{\frac{\rho}{k}}-2\left(1-\mu_{1}\right) \alpha_{1} \mathrm{e}^{-\lambda \sqrt{\frac{\rho}{k}} \widehat{y}} \sinh \lambda \sqrt{\frac{\rho}{k}}\right]
$$

with

$$
\Delta_{1}^{\widehat{y}_{n, m}}(\lambda):=\frac{1}{\lambda}\left[-2 \alpha_{1} \mu_{1} \sinh \lambda \sqrt{\frac{\rho}{k}}-2 \sqrt{\rho k} \cosh \lambda \sqrt{\frac{\rho}{k}}-2\left(1-\mu_{1}\right) \alpha_{1} \mathrm{e}^{-\lambda \sqrt{\frac{\rho}{k}} \widehat{y}_{n, m}} \sinh \lambda \sqrt{\frac{\rho}{k}}\right]
$$

Suppose that $\lambda_{n, m}$ is a zero for $\Delta_{1}^{\widehat{y}_{n, m}}(\lambda)$ with positive real part and given by (5.1). Then for $\left|\lambda-\lambda_{n, m}\right| \leq \frac{1}{2} \Re \lambda_{n, m}$, using Taylor formula, we have

$$
\left|\Delta_{1}^{\widehat{y}}(\lambda)-\Delta_{1}^{\widehat{y}_{n, m}}(\lambda)\right|=2\left(1-\mu_{1}\right) \alpha_{1}\left|\lambda^{-1}\right|\left|\mathrm{e}^{-\lambda \sqrt{\frac{\rho}{k}} \widehat{y}_{n, m}}-\mathrm{e}^{-\lambda \sqrt{\frac{\rho}{k}} \widehat{y}}\right|\left|\sinh \lambda \sqrt{\frac{\rho}{k}}\right| \leq\left|\Delta_{1}^{\widehat{y}_{n, m}}(\lambda)\right| .
$$

Hence, the Rouché theorem says that $\Delta_{1}^{\widehat{y}_{n, m}}(\lambda)$ and $\Delta_{1}^{\widehat{y}}(\lambda)$ have the same number of zeros in $\left|\lambda-\lambda_{n, m}\right| \leq \frac{1}{2} \Re \lambda_{n, m}$. Since $\Delta_{1}^{\widehat{y}_{n, m}}(\lambda)$ has at least one zero with positive real part, the same is true for $\Delta_{1}^{\widehat{y}}(\lambda)$ which implies that $\Delta_{1}(\lambda)$ has zeros with positive real part. Let $n$ in (5.1) be sufficiently large. By the Rouché theorem again, there exists at least one eigenvalue of $\mathcal{A}$ with positive real part. Therefore, the system (1.7) is unstable.

For the case $\mu_{2}<\frac{1}{2}$, by the similar discussion, there exists at least one eigenvalue of $\mathcal{A}$ with positive real part which also lead to the instability of the system (1.7). The proof is complete.

Now, let us consider the stability of the system (1.7) when $\mu_{i} \geq \frac{1}{2}, i=1,2$. Firstly, from [18], we introduce the fundamental solution to (3.2) as follows

$$
\begin{aligned}
w(x) & =w(0) w_{1}(x, \lambda)+\varphi(0) w_{2}(x, \lambda)+w_{x}(0) w_{3}(x, \lambda)+\varphi_{x}(0) w_{4}(x, \lambda) \\
\varphi(x) & =w(0) \varphi_{1}(x, \lambda)+\varphi(0) \varphi_{2}(x, \lambda)+w_{x}(0) \varphi_{3}(x, \lambda)+\varphi_{x}(0) \varphi_{4}(x, \lambda)
\end{aligned}
$$


The expressions of $w_{i}, \varphi_{i}, i=1,2,3,4$ can be gotten directly from [18] and given as follows

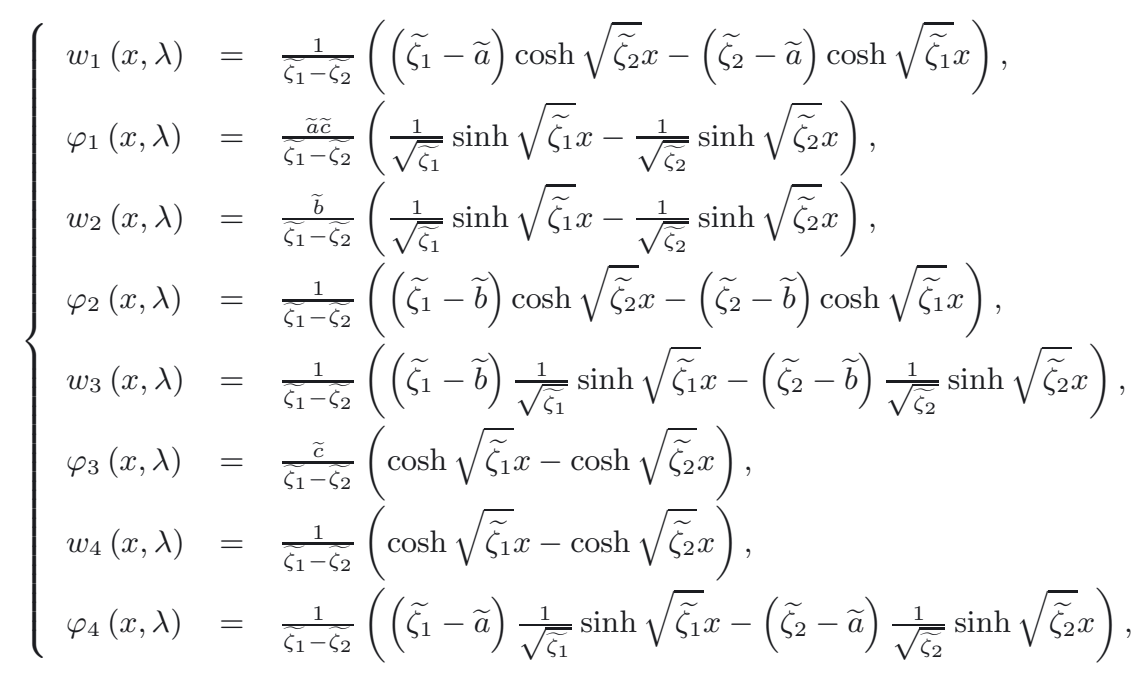

where

$$
\widetilde{a}=\frac{\rho}{k} \lambda^{2}, \widetilde{b}=\frac{I_{\rho}}{E I} \lambda^{2}+\frac{k}{E I}, \widetilde{c}=-\frac{k}{E I},
$$

and $\widetilde{\zeta}_{i}, i=1,2$ are the two roots of the following quadratic equation:

$$
\widetilde{\zeta}^{2}-(\widetilde{a}+\widetilde{b}+\widetilde{c}) \widetilde{\zeta}+\widetilde{a} \widetilde{b}=0
$$

We have the following result:

Theorem 5.2. Let $\mathcal{A}$ and $\mathcal{H}$ be defined as before. Then,

(1) when $\mu_{1}>\frac{1}{2}, \mu_{2}>\frac{1}{2}$, the system (1.7) is asymptotically stable;

(2) when $\mu_{1}=\frac{1}{2}, \mu_{2}>\frac{1}{2}$, if there exists $k \in \mathbb{Z}$ such that the vectors

$$
\left(w_{j x}\left(1, \frac{(2 k+1) \pi i}{-\tau_{1}}\right), \varphi_{j x}\left(1, \frac{(2 k+1) \pi i}{-\tau_{1}}\right), \varphi_{j}\left(1, \frac{(2 k+1) \pi i}{-\tau_{1}}\right)\right)^{\tau}, \quad j=3,4
$$

are linearly dependent, then the system (1.7) is unstable; otherwise, this system is asymptotically stable;

(3) when $\mu_{1}>\frac{1}{2}, \mu_{2}=\frac{1}{2}$, if there exists $k \in \mathbb{Z}$ such that

$$
\left(w_{j x}\left(1, \frac{(2 k+1) \pi i}{-\tau_{2}}\right)-\varphi_{j}\left(1, \frac{(2 k+1) \pi i}{-\tau_{2}}\right), \varphi_{j x}\left(1, \frac{(2 k+1) \pi i}{-\tau_{2}}\right), w_{j}\left(1, \frac{(2 k+1) \pi i}{-\tau_{2}}\right)\right)^{\tau}, \quad j=3,4
$$

are linearly dependent, the system (1.7) is unstable; otherwise, this system is asymptotically stable.

Proof. When $\mu_{1} \geq \frac{1}{2}, \mu_{2} \geq \frac{1}{2}$, from Section 2, we get that $\mathcal{A}$ is dissipative in $\mathcal{H}$. Therefore, according to the Lyubich and Phóng theorem [6], we only need to verify that there is no eigenvalue of $\mathcal{A}$ on the imaginary axis to get the asymptotic stability of the system (1.7).

If there exists $\sigma \in \mathbb{R}$ such that $\lambda=i \sigma$ is an eigenvalue of $\mathcal{A}$ and $W=(w, \lambda w, p, \varphi, \lambda \varphi, q)^{\tau}$ is an eigenvector of $\mathcal{A}$ corresponding to $\lambda$, then we have

$$
0=\Re \lambda(W, W)_{1}=\Re(\mathcal{A} W, W)_{1} .
$$

We consider the asymptotic stability of the system (1.7) under these following cases. 
Case 1: $\mu_{1}>\frac{1}{2}, \mu_{2}>\frac{1}{2}$

From (2.11) and (5.7), we get $p(0)=q(0)=p(1)=q(1)=0$. Hence, according to (3.1), we have that $\lambda=i \sigma$ satisfy

$$
\left\{\begin{array}{l}
\rho \lambda^{2} w-k\left(w_{x x}-\varphi_{x}\right)=0, \quad x \in(0,1) \\
I_{\rho} \lambda^{2} \varphi-E I \varphi_{x x}-k\left(w_{x}-\varphi\right)=0, \quad x \in(0,1) \\
w(0)=\varphi(0)=w(1)=\varphi(1)=0, \quad w_{x}(1)=\varphi_{x}(1)=0
\end{array}\right.
$$

Therefore, $w(1)=\varphi(1)=w_{x}(1)=\varphi_{x}(1)=0$ together with the fundamental solution (5.2)-(5.6) implies that

$$
w(x)=\varphi(x)=0 .
$$

Thus, $(w, \lambda w, p, \varphi, \lambda \varphi, q)^{\tau}=0$ which contradicts the assumption that $\lambda=i \sigma$ is an eigenvalue of $\mathcal{A}$. So there is no eigenvalue on the imaginary axis. The Lyubich and Phóng theorem [6] asserts that the system (1.7) is asymptotically stable.

Case 2: $\mu_{1}=\frac{1}{2}, \mu_{2}>\frac{1}{2}$.

Similarly, if $\lambda=i \sigma$ is an eigenvalue of $\mathcal{A}$, we can get

$$
\begin{aligned}
0= & 2 \Re(\mathcal{A} W, W)_{1} \\
= & -2\left(\alpha_{1} \mu_{1}-\frac{\eta_{1}}{2 \tau_{1}}\right) p(0) \overline{p(0)}-2\left(\alpha_{2} \mu_{2}-\frac{\eta_{2}}{2 \tau_{2}}\right) q(0) \overline{q(0)}-\frac{\eta_{1}}{\tau_{1}} p(1) \overline{p(1)}-\frac{\eta_{2}}{\tau_{2}} q(1) \overline{q(1)} \\
& -\alpha_{1}\left(1-\mu_{1}\right) p(0) \overline{p(1)}-\alpha_{1}\left(1-\mu_{1}\right) p(1) \overline{p(0)}-\alpha_{2}\left(1-\mu_{2}\right) q(0) \overline{q(1)}-\alpha_{2}\left(1-\mu_{2}\right) q(1) \overline{q(0)} .
\end{aligned}
$$

Since (2.9) implies that $\eta_{1}=\frac{\tau_{1} \alpha_{1}}{2}$

$$
\begin{aligned}
2 \Re(\mathcal{A} W, W)_{1}= & -\frac{\alpha_{1}}{2}\left(p(0)+p(1) \overline{(p(0)+p(1))}-2\left(\alpha_{2} \mu_{2}-\frac{\eta_{2}}{2 \tau_{2}}\right) q(0) \overline{q(0)}\right. \\
& -\frac{\eta_{2}}{\tau_{2}} q(1) \overline{q(1)}-\alpha_{2}\left(1-\mu_{2}\right) q(0) \overline{q(1)}-\alpha_{2}\left(1-\mu_{2}\right) q(1) \overline{q(0)} \\
\leq & -\frac{\alpha_{1}}{2}\left(p(0)+p(1) \overline{(p(0)+p(1))}-2\left(\alpha_{2} \mu_{2}-\frac{\eta_{2}}{2 \tau_{2}}-\frac{\alpha_{2}\left(1-\mu_{2}\right)}{2}\right)|q(0)|^{2}\right. \\
& -2\left(\frac{\eta_{2}}{2 \tau_{2}}-\frac{\alpha_{2}\left(1-\mu_{2}\right)}{2}\right)|q(1)|^{2} .
\end{aligned}
$$

From (5.7), we get

$$
p(0)+p(1)=0, \quad q(0)=\lambda \varphi(1)=0, \quad q(1)=0 .
$$

This implies that $\lambda=i \sigma$ is an eigenvalue if and only if the following equation has nonzero solution:

$$
\left\{\begin{array}{l}
\rho \lambda^{2} w-k\left(w_{x x}-\varphi_{x}\right)=0, \quad x \in(0,1) \\
I_{\rho} \lambda^{2} \varphi-E I \varphi_{x x}-k\left(w_{x}-\varphi\right)=0, \quad x \in(0,1) \\
\tau_{1} \lambda p(x)+p_{x}(x)=0, \quad \tau_{2} \lambda q(x)+q_{x}(x)=0 \\
w(0)=\varphi(0)=\varphi(1)=0 \\
k\left(w_{x}-\varphi\right)(1)=-\frac{\alpha_{1}}{2}(\lambda w(1)+p(1))=0, \quad E I \varphi_{x}(1)=0
\end{array}\right.
$$

Then, using the fundamental solution to Timoshenko beam (5.2)-(5.6), we have

$$
w=c w_{3}(x, \lambda)+d w_{4}(x, \lambda), \quad \varphi=c \varphi_{3}(x, \lambda)+d \varphi_{4}(x, \lambda),
$$

where $c=w_{x}(0)$ and $d=\varphi_{x}(0)$. By (5.9) and the third equation in (5.10), a direct calculation yields

$$
p(0)\left(1+\mathrm{e}^{-\tau_{1} \lambda}\right)=0 .
$$

From the proof in Case 1, we know that if $p(0)=0$, then $\lambda=i \sigma \notin \sigma(\mathcal{A})$. Hence, if $\lambda=i \sigma$ is an eigenvalue of $\mathcal{A}$, it must satisfy $1+\mathrm{e}^{-\tau_{1} \lambda}=0$, which implies

$$
\lambda=\frac{(2 k+1) \pi i}{-\tau_{1}}, \quad k \in \mathbb{Z}
$$


Then, substituting (5.11) and (5.12) into the boundary conditions in (5.10) yields

$$
\left[\begin{array}{cc}
w_{3 x}\left(1, \frac{(2 k+1) \pi i}{-\tau_{1}}\right) & w_{4 x}\left(1, \frac{(2 k+1) \pi i}{-\tau_{1}}\right) \\
\varphi_{3 x}\left(1, \frac{(2 k+1) \pi i}{-\tau_{1}}\right) & \varphi_{4 x}\left(1, \frac{(2 k+1) \pi i}{-\tau_{1}}\right) \\
\varphi_{3}\left(1, \frac{(2 k+1) \pi i}{-\tau_{1}}\right) & \varphi_{4}\left(1, \frac{(2 k+1) \pi i}{-\tau_{1}}\right)
\end{array}\right]\left[\begin{array}{l}
c \\
d
\end{array}\right]=0 .
$$

Therefore, (5.10) has nonzero solution if and only if there exists $k \in \mathbb{Z}$ such that the vectors $\left(w_{j x}\left(1, \frac{(2 k+1) \pi i}{-\tau_{1}}\right)\right.$, $\left.\varphi_{j x}\left(1, \frac{(2 k+1) \pi i}{-\tau_{1}}\right), \varphi_{j}\left(1, \frac{(2 k+1) \pi i}{-\tau_{1}}\right)\right)^{\tau}, j=3,4$ are linearly dependent, which leads to the instability of the system (1.7). Otherwise, the system (1.7) is asymptotically stable.

Case 3: $\mu_{1}>\frac{1}{2}, \mu_{2}=\frac{1}{2}$.

If $\lambda=i \sigma$ is an eigenvalue of $\mathcal{A}$, we have

$$
p(0)=p(1)=0, \quad q(0)+q(1)=0 .
$$

Similarly with the proof in Case 2 , we have that if there exists $k \in \mathbb{Z}$ such that $\left(w_{j x}\left(1, \frac{(2 k+1) \pi i}{-\tau_{2}}\right)-\varphi_{j}\left(1, \frac{(2 k+1) \pi i}{-\tau_{2}}\right)\right.$, $\left.\varphi_{j x}\left(1, \frac{(2 k+1) \pi i}{-\tau_{2}}\right), w_{j}\left(1, \frac{(2 k+1) \pi i}{-\tau_{2}}\right)\right)^{\tau}, j=3,4$ are linearly dependent, the system (1.7) is unstable. Otherwise, this system is asymptotically stable. The proof is complete.

Remark 5.1. " $\mu_{1}=\mu_{2}=\frac{1}{2}$ " is a critical point for the stability of the system (1.7). Under this condition, the stability of the system (1.7) is very complicated. It can be divided into the following four cases:

(a) $p(0)=q(0)=0$.

According to Case 1 in Theorem 5.2, the system is asymptotically stable.

(b) $q(0)=1+\mathrm{e}^{-\tau_{1} \lambda}=0$.

From Case 2 in Theorem 5.2, if there exists $k \in \mathbb{Z}$ such that vectors $\left(w_{j x}\left(1, \frac{(2 k+1) \pi i}{-\tau_{1}}\right), \varphi_{j x}\left(1, \frac{(2 k+1) \pi i}{-\tau_{1}}\right)\right.$, $\left.\varphi_{j}\left(1, \frac{(2 k+1) \pi i}{-\tau_{1}}\right)\right)^{\tau}, j=3,4$ are linearly dependent, the system (1.7) is unstable. Otherwise, this system is asymptotically stable.

(c) $p(0)=1+\mathrm{e}^{-\tau_{2} \lambda}=0$.

By Case 3 in Theorem 5.2, if there exists $k \in \mathbb{Z}$ such that $\left(w_{j x}\left(1, \frac{(2 k+1) \pi i}{-\tau_{2}}\right)-\varphi_{j}\left(1, \frac{(2 k+1) \pi i}{-\tau_{2}}\right), \varphi_{j x}\left(1, \frac{(2 k+1) \pi i}{-\tau_{2}}\right)\right.$, $\left.w_{j}\left(1, \frac{(2 k+1) \pi i}{-\tau_{2}}\right)\right)^{\tau}, j=3,4$ are linearly dependent, the system (1.7) is unstable. Otherwise, this system is asymptotically stable.

(d) $1+\mathrm{e}^{-\tau_{1} \lambda}=1+\mathrm{e}^{-\tau_{2} \lambda}=0$.

A direct calculation leads to $\lambda=i \sigma=\frac{i(2 m+1) \pi}{-\tau_{1}}=\frac{i(2 n+1) \pi}{-\tau_{2}}, m, n \in \mathbb{Z}$. From (3.1) and (5.7), we also find that this $\lambda=i \sigma$ must satisfy $k\left(w_{x}-\varphi\right)(1)=E I \varphi_{x}(1)=0$. By the fundamental solution to Timoshenko beam, it yields

$$
\widetilde{\Delta}(\lambda):=\left|\begin{array}{cc}
w_{3 x}(1, \lambda)-\varphi_{3}(1, \lambda) & w_{4 x}(1, \lambda)-\varphi_{4}(1, \lambda) \\
\varphi_{3 x}(1, \lambda) & \varphi_{4 x}(1, \lambda)
\end{array}\right|=0
$$

Therefore, if $\frac{\tau_{1}}{\tau_{2}}$ is a rational number and $\widetilde{\Delta}\left(\frac{\left(2 m_{j}+1\right) \pi i}{-\tau_{j}}\right)=0$ for some $m_{j} \in \mathbb{Z}, j=1,2$, the system (1.7) is unstable. Otherwise, this system is asymptotically stable.

In order to discuss the exponential stability of the system (1.7), we have the following result: 
Lemma 5.1. Let $\mathcal{H}$ and $\mathcal{A}$ be defined as before and the conditions (2.8), (3.14) be fulfilled. Then the system (1.7) is exponentially stable if and only if

$$
\inf _{\lambda \in i \mathbb{R}}|\Delta(\lambda)|>0
$$

Proof. Necessity: Under the condition (2.8), we know that $\mathcal{A}$ generates a $C_{0}$ semigroup of contractions on $\mathcal{H}$, which implies that there is no eigenvalue of $\mathcal{A}$ on the right half complex plane. If $\inf _{\lambda \in i \mathbb{R}}|\Delta(\lambda)|=0$, there always exists a sequence $\lambda_{j}, j=1,2, \ldots, \Re \lambda_{j} \rightarrow 0, \Im \lambda_{j} \rightarrow \infty$ such that $\left|\Delta\left(\lambda_{j}\right)\right| \rightarrow 0, j \rightarrow \infty$, which implies that the imaginary axis is the asymptote of $\sigma(\mathcal{A})$. Since the system (1.7) satisfies spectrum determined growth condition, this system is not exponentially stable, which is a contradiction. Therefore, (5.15) holds.

Sufficiency: According to the proof of Necessity, it is easy to check that (5.15) implies that the imaginary axis is not an asymptote of $\sigma(\mathcal{A})$. Thus, the spectrum determined growth condition of the system (1.7) implies that this system is exponentially stable.

Based on this lemma, we get the following result about the exponential stability of the system (1.7).

Theorem 5.3. Let $\mathcal{A}$ and $\mathcal{H}$ be defined as before and the condition (3.14) be fulfilled. If $\mu_{1}>\frac{1}{2}, \mu_{2}>\frac{1}{2}$, then the closed loop system (1.7) is exponentially stable.

Proof. From Theorem 5.2, we know under the conditions $\mu_{1}>\frac{1}{2}, \mu_{2}>\frac{1}{2}$, the system (1.7) is asymptotically stable. From Lemma 5.1, we only need to check (5.15). To this end, set $\xi \in \mathbb{R}$. Since $\Delta(\lambda)=\Delta_{1}(\lambda) \Delta_{2}(\lambda)+$ $\mathcal{O}\left(\lambda^{-1}\right)$, it only needs to show that

$$
\Delta_{j}(i \xi) \neq 0, \quad \xi \rightarrow \infty, j=1,2
$$

where $\Delta_{j}(\lambda), j=1,2$ are given by (3.23) and (3.24). A direct calculation leads to

$$
\begin{aligned}
\Delta_{1}(\lambda)= & 2 i\left[-\alpha_{1} \mu_{1} \sin \sqrt{\frac{\rho}{k}} \xi-\alpha_{1}\left(1-\mu_{1}\right) \cos \tau_{1} \xi \sin \sqrt{\frac{\rho}{k}} \xi\right]+2\left[-\alpha_{1}\left(1-\mu_{1}\right) \sin \tau_{1} \xi \sin \sqrt{\frac{\rho}{k}} \xi\right. \\
& \left.-\sqrt{\rho k} \cos \sqrt{\frac{\rho}{k}} \xi\right], \\
\Delta_{2}(\lambda)= & 2 i\left[-\alpha_{2} \mu_{2} \sin \sqrt{\frac{I_{\rho}}{E I}} \xi-\alpha_{2}\left(1-\mu_{2}\right) \cos \tau_{2} \xi \sin \sqrt{\frac{I_{\rho}}{E I}} \xi\right]+2\left[-\alpha_{2}\left(1-\mu_{2}\right) \sin \tau_{2} \xi \sin \sqrt{\frac{I_{\rho}}{E I}} \xi\right. \\
& \left.-\sqrt{I_{\rho} E I} \cos \sqrt{\frac{I_{\rho}}{E I}} \xi\right] .
\end{aligned}
$$

Then $\Delta_{1}(i \xi)=0$ is equivalent to

$$
\begin{aligned}
& \alpha_{1} \mu_{1} \sin \sqrt{\frac{\rho}{k}} \xi+\alpha_{1}\left(1-\mu_{1}\right) \cos \tau_{1} \xi \sin \sqrt{\frac{\rho}{k}} \xi=0, \\
& \alpha_{1}\left(1-\mu_{1}\right) \sin \tau_{1} \xi \sin \sqrt{\frac{\rho}{k}} \xi+\sqrt{\rho k} \cos \sqrt{\frac{\rho}{k}} \xi=0 .
\end{aligned}
$$

Similarly, $\Delta_{2}(i \xi)=0$ is equivalent to

$$
\begin{gathered}
\alpha_{2} \mu_{2} \sin \sqrt{\frac{I_{\rho}}{E I}} \xi+\alpha_{2}\left(1-\mu_{2}\right) \cos \tau_{2} \xi \sin \sqrt{\frac{I_{\rho}}{E I}} \xi=0, \\
\alpha_{2}\left(1-\mu_{2}\right) \sin \tau_{2} \xi \sin \sqrt{\frac{I_{\rho}}{E I}} \xi+\sqrt{I_{\rho} E I} \cos \sqrt{\frac{I_{\rho}}{E I}} \xi=0 .
\end{gathered}
$$


Now we show $\Delta_{1}(i \xi) \neq 0, \xi \rightarrow \infty$ in the following two cases:

Case 1: $\mu_{1}=1$.

Under this condition, (5.16) and (5.17) imply that $\sin \sqrt{\frac{\rho}{k}} \xi=\cos \sqrt{\frac{\rho}{k}} \xi=0$, which is a contradiction. Thus, $\Delta_{1}(i \xi) \neq 0$ for any $\xi \in \mathbb{R}, \xi \rightarrow \infty$.

Case 2: $\frac{1}{2}<\mu_{1}<1$.

From (5.16), we have $\alpha_{1} \mu_{1}+\alpha_{1}\left(1-\mu_{1}\right) \cos \tau_{1} \xi=0$ or $\sin \sqrt{\frac{\rho}{k}} \xi=0$. It is easy to check that $\sin \sqrt{\frac{\rho}{k}} \xi \neq 0$. Hence, we can get

Substituting (5.20) into (5.17) leads to

$$
\cos \tau_{1} \xi=-\frac{\mu_{1}}{1-\mu_{1}}
$$

$$
\pm \alpha_{1}\left(1-\mu_{1}\right) \sqrt{1-\left(\frac{-\mu_{1}}{1-\mu_{1}}\right)^{2}} \sin \sqrt{\frac{\rho}{k}} \xi-\sqrt{\rho k} \cos \sqrt{\frac{\rho}{k}} \xi=0 .
$$

From (5.16) and (5.17), we have $\cos \sqrt{\frac{\rho}{k}} \xi \neq 0$. Therefore,

$$
\tan \sqrt{\frac{\rho}{k}} \xi= \pm \frac{\sqrt{\rho k}}{\alpha_{1}\left(1-\mu_{1}\right) \sqrt{1-\left(\frac{\mu_{1}}{1-\mu_{1}}\right)^{2}}}= \pm \frac{\sqrt{\rho k}}{\alpha_{1} \sqrt{1-2 \mu_{1}}} .
$$

If $\frac{1}{2}<\mu_{1}<1, \frac{\sqrt{\rho k}}{\alpha_{1} \sqrt{1-2 \mu_{1}}}$ is an imaginary number, which contradicts the fact that $\tan \sqrt{\frac{\rho}{k}} \xi \in \mathbb{R}$ for $\xi \in \mathbb{R}$. Therefore, $\Delta_{1}(i \xi) \neq 0$ for any $\xi \in \mathbb{R}, \xi \rightarrow \infty$ when $\mu_{1} \neq 1$.

By the similar method, we get $\Delta_{2}(i \xi) \neq 0$ for any $\xi \in \mathbb{R}, \xi \rightarrow \infty$ when $\mu_{2}>\frac{1}{2}$. Based on the discussion above, by Lemma 5.1, the system (1.7) is exponentially stable. The proof is complete.

When $\mu_{1}=\frac{1}{2}$ or $\mu_{2}=\frac{1}{2}$, from Theorem 5.2 and Remark 5.1, the system (1.7) can be asymptotically stable, based on which this system is only exponentially stable under strict conditions.

Theorem 5.4. Let $\mathcal{A}$ and $\mathcal{H}$ be defined as before and the conditions (2.8), (3.14) be fulfilled. Suppose that the system (1.7) is asymptotically stable. Then

if $\mu_{1}=\frac{1}{2}, \mu_{2}>\frac{1}{2}$ and $\sqrt{\frac{\rho}{k}}=\tau_{1}$, the system (1.7) is exponentially stable;

if $\mu_{1}>\frac{1}{2}, \mu_{2}=\frac{1}{2}$ and $\sqrt{\frac{I_{\rho}}{E I}}=\tau_{2}$, the system (1.7) is exponentially stable;

if $\mu_{1}=\frac{1}{2}, \mu_{2}=\frac{1}{2}$ and $\sqrt{\frac{\rho}{k}}=\tau_{1}, \sqrt{\frac{I_{\rho}}{E I}}=\tau_{2}$, the system (1.7) is exponentially stable.

Proof. When $\mu_{1}=\frac{1}{2}$, from (5.16) and (5.17), $\Delta_{1}(i \xi)=0$ is equivalent to

$$
\begin{aligned}
& \frac{1}{2} \alpha_{1} \sin \sqrt{\frac{\rho}{k}} \xi+\frac{1}{2} \alpha_{1} \cos \tau_{1} \xi \sin \sqrt{\frac{\rho}{k}} \xi=0, \\
& \frac{1}{2} \alpha_{1} \sin \tau_{1} \xi \sin \sqrt{\frac{\rho}{k}} \xi+\sqrt{\rho k} \cos \sqrt{\frac{\rho}{k}} \xi=0 .
\end{aligned}
$$

A direct calculation yields

$$
\sin \sqrt{\frac{\rho}{k}} \xi \neq 0, \quad \frac{1}{2} \alpha_{1}+\frac{1}{2} \alpha_{1} \cos \tau_{1} \xi=\sin \tau_{1} \xi=\cos \sqrt{\frac{\rho}{k}} \xi=0, \xi \in \mathbb{R} .
$$

Then, if $\sqrt{\frac{\rho}{k}}=\tau_{1}$, we can not find a sequence $\xi_{n} \in \mathbb{R}$, such that $\sin \tau_{1} \xi_{n} \rightarrow 0, \cos \sqrt{\frac{\rho}{k}} \xi_{n} \rightarrow 0$ simultaneously, which leads to $\inf _{\xi \in \mathbb{R}}\left|\Delta_{1}(i \xi)\right| \neq 0$. Similarly, when $\mu_{2}=\frac{1}{2}$ we obtain that if $\sqrt{\frac{I_{\rho}}{E I}}=\tau_{2}$, then $\inf _{\xi \in \mathbb{R}}\left|\Delta_{2}(i \xi)\right| \neq 0$. 


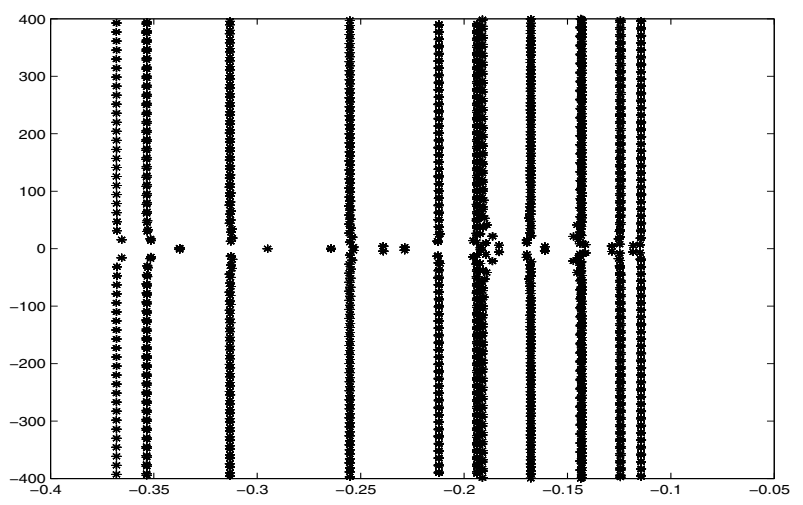

(a) $\mu_{1}=0.8, \mu_{2}=0.6$

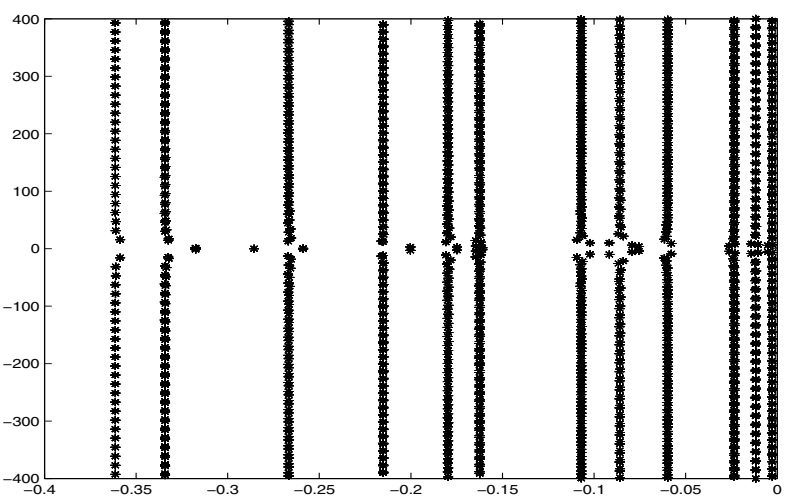

(b) $\mu_{1}=0.5, \mu_{2}=0.5$

Figure 1. Distribution of the spectrum.

We have known from the proof of Theorem 5.3 that if $\mu_{j}>\frac{1}{2}$, then $\Delta_{j}(i \xi) \neq 0, \xi \rightarrow \infty, \xi \in \mathbb{R}$ holds for each $j, j=1,2$. Thus, if $\mu_{1}=\frac{1}{2}, \mu_{2}>\frac{1}{2}$ and $\sqrt{\frac{\rho}{k}}=\tau_{1}$,

$$
\inf _{\xi \in \mathbb{R}}|\Delta(i \xi)|=\inf _{\xi \in \mathbb{R}}\left|\Delta_{1}(i \xi) \Delta_{2}(i \xi)\right|>0 .
$$

Therefore, Lemma 5.1 asserts that the system (1.7) is exponentially stable.

Similarly, we can get that if $\mu_{1}>\frac{1}{2}, \mu_{2}=\frac{1}{2}$ and $\sqrt{\frac{I_{\rho}}{E I}}=\tau_{2}$, the system (1.7) is exponentially stable as well.

If $\mu_{1}=\frac{1}{2}, \mu_{2}=\frac{1}{2}$, under the conditions $\sqrt{\frac{\rho}{k}}=\tau_{1}, \sqrt{\frac{I_{\rho}}{E I}}=\tau_{2}$, the system (1.7) is also exponentially stable by the similar discussion. The proof is complete.

\section{Simulations}

In this section, we shall give some simulations of the system (1.7) to support our results. Firstly, we give each parameter in this system a numerical value as follows:

\begin{tabular}{|c|c|c|c|c|c|c|c|}
\hline$\rho$ & $k$ & $I_{\rho}$ & $E I$ & $\tau_{1}$ & $\tau_{2}$ & $\alpha_{1}$ & $\alpha_{2}$ \\
\hline 27 & 3 & 8 & 2 & 0.5 & 0.4 & 5 & 6 \\
\hline
\end{tabular}

Then let us discuss the distribution of the spectrum of $\mathcal{A}$ under the system parameters chosen above. Based on the fundamental solution to Timoshenko beams (5.2)-(5.6) and the Matlab scientific calculation, we get many simulations for the distribution of the spectrum of $\mathcal{A}$ by changing the value of $\mu_{i}, i=1,2$. We find that in these simulations the eigenvalues of $\mathcal{A}$ distribute in conjugate pairs in the complex plane and there are many "vertical" lines which the eigenvalues approach. These lines are in fact the asymptotes of the eigenvalues of $\mathcal{A}$. Here we only show two figures. Figures 1(a) and 1(b) denote the distribution of the spectrum of $\mathcal{A}$ when $\mu_{1}=0.8, \mu_{2}=0.6$ and $\mu_{1}=0.5, \mu_{2}=0.5$, respectively, in which "* " denotes the eigenvalues of $\mathcal{A}$.

We find that if we choose $\mu_{i}>\frac{1}{2}, i=1,2$, the eigenvalues of $\mathcal{A}$ are always located in the left hand of the complex plane and far away from the imaginary axis, which implies the exponential stability of the system. We see in Figure 1(a) that the eigenvalues of $\mathcal{A}$ satisfy that $\Re \lambda<-0.1$. Since the spectrum determined growth condition holds, this system is exponentially stable when $\mu_{1}=0.8, \mu_{2}=0.6$. Furthermore, the exponential decay rate is less than -0.1 .

However, when $\mu_{i} \rightarrow 0.5, i=1,2$, the distance between the maximum of the real part of the eigenvalues and the imaginary axis becomes smaller, which implies that the system (1.7) decays more slowly when $\mu_{i} \rightarrow 0.5, i=1,2$. Furthermore, when $\mu_{1}=0.5, \mu_{2}=0.5$, we see in Figure 1 (b) that there is no vertical line to divide the spectrum and the imaginary axis, which shows that the imaginary axis may be the asymptote 
of the eigenvalues of $\mathcal{A}$. Therefore, the system (1.7) is possibly asymptotically stable but not exponentially stable when $\mu_{i}=0.5, i=1,2$.

Acknowledgements. The authors would like to thank the referees for their useful and helpful comments and suggestions.

\section{REFERENCES}

[1] J.W. Brown and R.V. Churchill, Complex variables and applications. Seventh Edition, China Machine Press, Beijing (2004).

[2] R. Datko, Two examples of ill-posedness with respect to small time delays in stabilized elastic systems. IEEE Trans. Automat. Contr. 38 (1993) 163-166.

[3] J.U. Kim and Y. Renardy, Boundary control of the Timoshenko beam. SIAM J. Control Optim. 25 (1987) 1417-1429.

[4] W.H. Kwon, G.W. Lee and S.W. Kim, Performance improvement, using time delays in multi-variable controller design. Int. J. Control 52 (1990) 1455-1473.

[5] J.S. Liang, Y.Q. Chen and B.Z. Guo, A new boundary control method for beam equation with delayed boundary measurement using modified smith predictors, in Proceedings of the 42nd IEEE Conference on Decision and Control, Hawaii (2003) 809-814.

[6] Yu.I. Lyubich and V.Q. Phóng, Asymptotic stability of linear differential equations in Banach spaces. Studia Math. 88 (1988) $34-37$.

[7] R. Mennicken and M. Möller, Non-self-adjoint boundary eigenvalue problem, North-Holland Mathematics Studies 192. NorthHolland, Amsterdam (2003).

[8] W. Michiels and S.I. Niculescu, Stability and stabilization of time-delay systems: An Eigenvalue-based approach. Society for Industrial and Applied Mathematics, Philadelphia (2007).

[9] O. Mörgul, On the stabilization and stability robustness against small delays of some damped wave equation. IEEE Trans. Automat. Contr. 40 (1995) 1626-1630.

[10] S. Nicaise and C. Pignotti, Stability and instability results of the wave equation with a delay term in the boundary or internal feedbacks. SIAM J. Control Optim. 45 (2006) 1561-1585.

[11] S. Nicaise and C. Pignotti, Stabilization of the wave equation with boundary or internal distributed delay. Differential and Integral Equations 21 (2008) 935-958.

[12] S. Nicaise and J. Valein, Stabilization of the wave equation on 1-D networks with a delay term in the nodal feedbacks. NHM 2 (2007) 425-479.

[13] A. Pazy, Semigroups of linear operators and applications to partial differential equations. Springer-Verlag, Berlin (1983).

[14] K. Sriram and M.S. Gopinathan, A two variable delay model for the circadian rhythm of Neurospora crassa. J. Theor. Biol. 231 (2004) 23-38.

[15] J. Srividhya and M.S. Gopinathan, A simple time delay model for eukaryotic cell cycle. J. Theor. Biol. 241 (2006) 617-627.

[16] H. Suh and Z. Bien, Use of time-delay actions in the controller design. IEEE Trans. Automat. Contr. 25 (1980) 600-603.

[17] S. Timoshenko, Vibration Problems in Engineering. Van Norstrand, New York (1955).

[18] Q.P. Vu, J.M. Wang, G.Q. Xu and S.P. Yung, Spectral analysis and system of fundamental solutions for Timoshenko beams. Appl. Math. Lett. 18 (2005) 127-134.

[19] G.Q. Xu and D.X. Feng, The Riesz basis property of a Timoshenko beam with boundary feedback and application. IMA J. Appl. Math. 67 (2002) 357-370.

[20] G.Q. Xu and B.Z. Guo, Riesz basis property of evolution equations in Hilbert space and application to a coupled string equation. SIAM J. Control Optim. 42 (2003) 966-984.

[21] G.Q. Xu and J.G. Jia, The group and Riesz basis properties of string systems with time delay and exact controllability with boundary control. IMA J. Math. Control Inf. 23 (2006) 85-96.

[22] G.Q. Xu and S.P. Yung, The expansion of semigroup and criterion of Riesz basis J. Differ. Equ. 210 (2005) 1-24.

[23] G.Q. Xu, Z.J. Han and S.P. Yung, Riesz basis property of serially connected Timoshenko beams. Int. J. Control 80 (2007) 470-485.

[24] G.Q. Xu, S.P. Yung and L.K. Li, Stabilization of wave systems with input delay in the boundary control. ESAIM: COCV 12 (2006) $770-785$.

[25] R.M. Young, An introduction to nonharmonic Fourier series. Academic Press, London (1980) 80-84. 